This item was submitted to Loughborough's Research Repository by the author.

Items in Figshare are protected by copyright, with all rights reserved, unless otherwise indicated.

\title{
Modelling and control of railway vehicle suspensions
}

PLEASE CITE THE PUBLISHED VERSION

PUBLISHER

(C) Springer

VERSION

AM (Accepted Manuscript)

LICENCE

CC BY-NC-ND 4.0

REPOSITORY RECORD

Zolotas, Argyrios C., and Roger M. Goodall. 2019. "Modelling and Control of Railway Vehicle Suspensions". figshare. https://hdl.handle.net/2134/4336. 
This item was submitted to Loughborough's Institutional Repository (https://dspace.lboro.ac.uk/) by the author and is made available under the following Creative Commons Licence conditions.

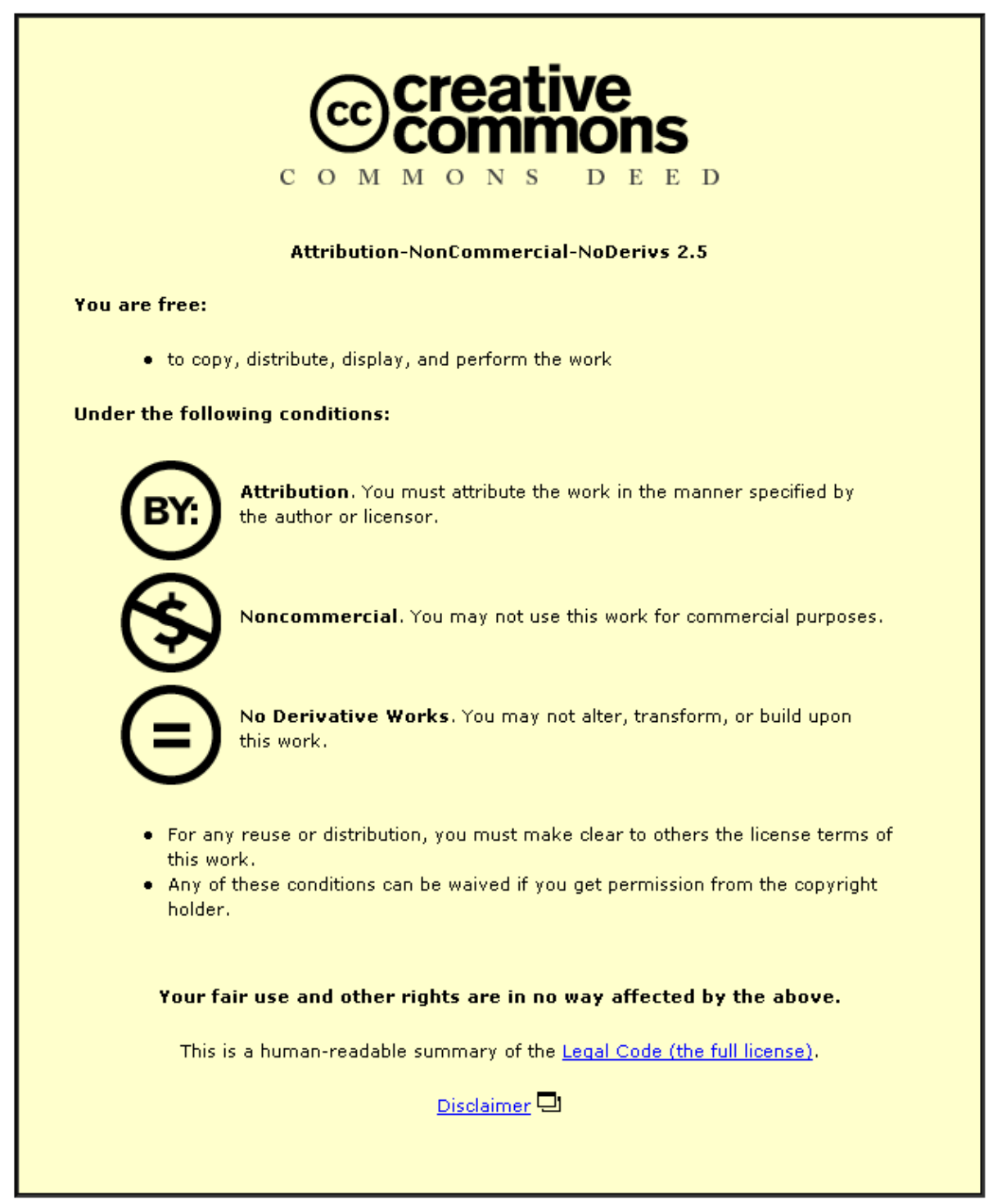

For the full text of this licence, please go to: http://creativecommons.org/licenses/by-nc-nd/2.5/ 


\title{
Modelling and Control of Railway Vehicle Suspensions
}

\author{
Argyrios C. Zolotas and Roger M. Goodall \\ Control Systems Group, Department of Electronic and Electrical Engineering, \\ Loughborough University, Loughborough, Leicestershire, LE11 2TJ, UK \\ a.zolotas@ieee.org \\ r.m.goodall@lboro.ac.uk
}

This chapter uses a railway vehicle as an example of a mechanical dynamic system to which control can be applied in a manner that yields significant benefits from an engineering and operational viewpoint. The first part describes the fundamentals of railway vehicles and their dynamics: the normal configuration, the suspension requirements, how they are modelled and an overview of the types of control concept that are currently applied or under consideration. The second part provides a case study of controller design issues.

\section{Overview of Railway Vehicle Dynamics and Control}

\subsection{Railway vehicles - conventional configuration}

Railway vehicles employ steel wheels running on tracks with steel rails, which provide the support and guidance functions. The interface between the two is established at contact point(s) between the wheels and rail surface, and both the vehicle configuration and the track greatly influence how vehicles behave $[1]$.

Most modern passenger-carrying railway vehicles have the configuration shown schematically in Fig. 1, which gives simplified side-view and end-view diagrams. The vehicle body is supported by two bogies using relatively soft secondary suspensions to provide isolation from the track-induced vibrations, i.e. to provide a good ride quality. Each bogie has four wheels arranged in two pairs, where each pair is rigidly connected via a common axle (known as the solid-axle wheelset) such that the two wheels have to rotate at the same speed. The wheelsets are connected to the bogie via primary suspension elements: these are much stiffer than in the secondary suspension and are designed to satisfy the vehicle's stability and guidance requirements. 


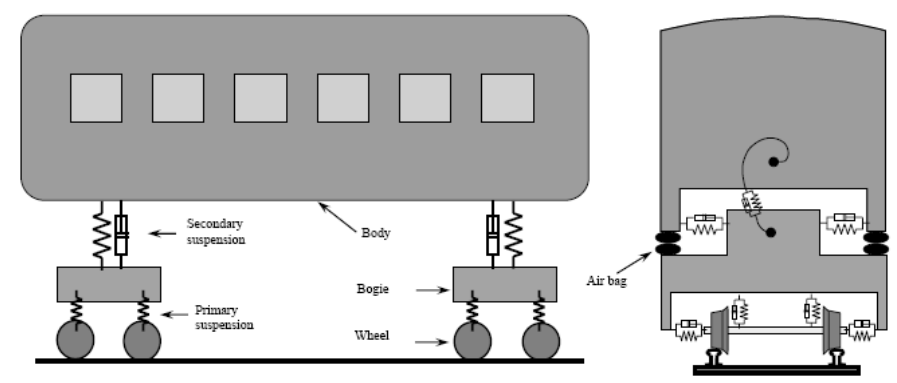

Fig. 1. Simplified side-view and end-view diagrams of a bogie vehicle

Both primary and secondary suspensions are provided in the vertical, lateral and longitudinal directions, and extra stiffness is often added in the secondary roll suspension. These suspensions mainly comprise passive springs and dampers connected in parallel and/or series, but airbags providing better performance are commonly used for the secondary suspension on modern passenger vehicles.

\subsection{Suspension design requirements}

Fundamentally there are three things that a suspension needs to do:

- support the (changing) weight of the vehicle

- provide guidance so that the vehicle follows the intended path

- provide isolation to give a satisfactory ride quality

The first two requirements are satisfied by a suspension that is relatively stiff, although in both cases this is a characteristic that is only really required at low frequencies, significantly less than $1 \mathrm{~Hz}$. The third requirement calls for a suspension that is soft, but the track irregularities that cause vibrations of the vehicle body don't become significant until around $1 \mathrm{~Hz}$ and higher. Hence there is clearly a design trade-off to be achieved in the suspension design. This trade-off is clarified by further consideration of the inputs.

The main dynamic excitations, in addition to the weight which changes rather slowly and is therefore essentially a quasi-static problem, are the track inputs, which can be divided into two types:

- Deterministic inputs

- Isolated features - steps, dips, short ramps, etc

- Intended inputs - gradients, curves, etc. having well-defined characteristics

Design requirement: constraint on suspension deflection

- Random inputs 
- Irregularities and imperfections (roughness of track)

- Characterised by a power spectrum (track velocity spectrum approximates to white noise)

Design requirement: satisfactory ride quality

In fact, designing any suspension is a non-trivial, multi-objective problem, particularly when combined with the dynamic complexity mentioned in the next subsection, and an active suspension brings additional issues. The overall process is summarised by Fig. 2. The inputs mentioned above are shown on the left, all of which occur in the vertical, lateral and roll direction.

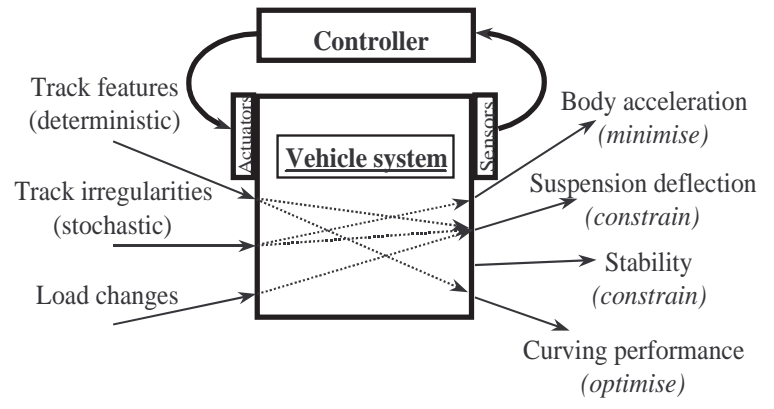

Fig. 2. Design requirements

The outputs are shown on the right, and four items are shown. The acceleration levels on the vehicle body, which represents the quality of ride, are to be minimised, and as mentioned the suspension movements must not become too large, i.e. they must be constrained. It is also necessary to ensure that there is a minimum margin of stability, a constraint which applies mainly to the wheelset dynamics determined by the primary suspension. The other primary suspension characteristic is its curving performance: essentially this is concerned with the guidance function, and is mainly about minimising any wear of the wheels and the rails, so an optimisation is needed. There are therefore three input types to consider, two output measures to minimise and two design constraints to meet, and as indicated for an active system it is necessary to choose a set of actuators, identify what measurements can practically be made to choose the set of sensors, then design a controller to satisfy the multiple objectives.

\subsection{Modelling of suspensions (for applying control)}

Railway vehicles are dynamically-complex multi-body systems. Each mass within the system has six dynamic degrees of freedom corresponding to three displacements (longitudinal, lateral and vertical) and three rotations (roll, pitch and yaw). Each degree of freedom results in a second-order differential 
equation and hence $6 \times N$ differential equations will be necessary to represent the system mathematically, where $N$ is the number of masses. For a conventional bogie vehicle, there are seven main masses (one vehicle body, two bogies and four wheelsets) and therefore a total of 42 second-order differential equations will be required if no constraints are considered. In addition, wheel-rail contact presents a highly non-linear dynamic/kinematic problem adding extra complexity to the already complex system.

In the modelling, Newton's law is applied to every degree of freedom of vehicle body and bogies, and external forces/torques are applied through suspension components. For design purposes, the suspensions can be largely considered as linear components, and can readily be generated using control design software such as Matlab. More thorough modelling for simulation will require the inclusion of non-linearities due to factors such as dead-band, hardening/softening and Coulomb friction elements, and nowadays will normally be undertaken using one of a number of 3D modelling packages which will incorporate the full complexity and non-linearity, including effects such as body flexibility that are essential for properly assessing ride quality, for example. It is of course essential that such modelling software can support the integration of the controller into the mechanical system.

The forces on the wheelset arise from so-called "creepages" between the wheel and rail, small relative velocities which arise because of elastic deformation of the steel at the point of contact and which apply in both the longitudinal and the lateral directions. Modelling of these effects is rather complex and non-linear [2], but it is a useful simplification to consider the effects separately for the two translational directions with linearized creep coefficients. Using this assumption, dynamic responses can be adequately described by deriving the creepages and using these coefficients to determine the corresponding creep forces generated at the contact interface.

For applying control to complex systems such as these, it is important to distinguish between the design model and the simulation model. The design model is a simplified version used for synthesis of the control strategy and algorithm, whereas the simulation model is a more complex version used to test fully the system performance. For example, there is a relatively weak coupling between the vertical and lateral motions of a vehicle and, depending on the objectives, only selected degrees of freedom need to be included in the design model. To study the vertical response, it would be adequate to include the bounce, pitch and sometimes roll degrees of freedom of the components. For the lateral response, the lateral, yaw and sometimes roll degrees of freedom are sufficient. In studies of the longitudinal dynamics, the longitudinal, pitch and roll degrees of freedom may be included in the model. As a common practice, the vehicle model is partitioned into side-view, plan-view and endview models. The side-view model is concerned with the bounce, pitch and longitudinal degrees of freedom; the plan-view model deals with the lateral and yaw motions and the end-view model covers the bounce and roll motions. 
The complete vehicle model can be assembled by determining which masses and directions of motion are to be included, and then creating a corresponding set of simultaneous differential equations that can be represented in matrix form.

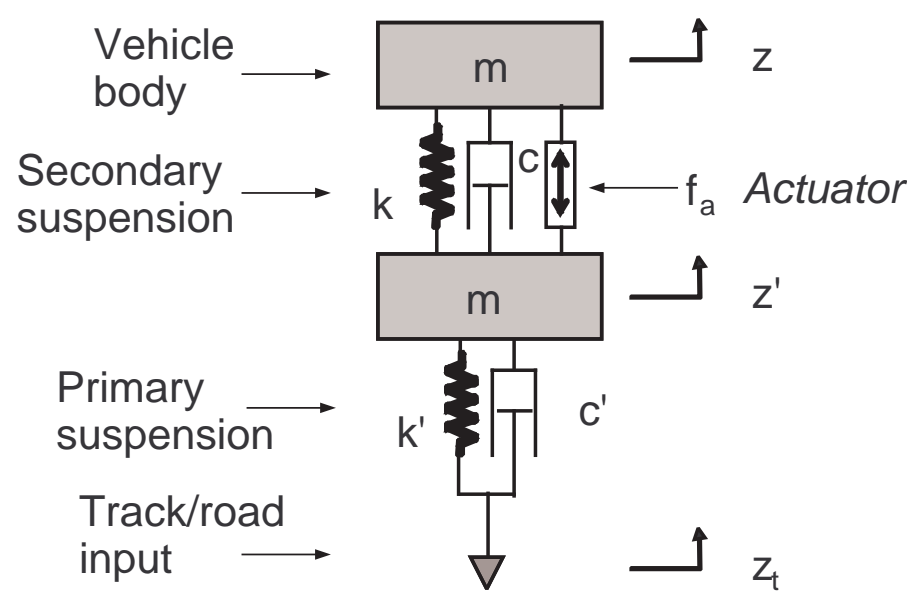

Fig. 3. Two-mass suspension example

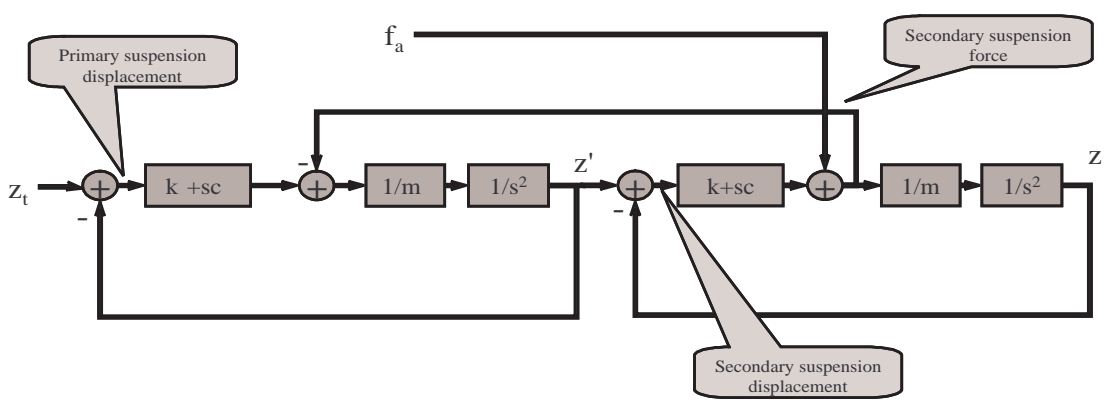

Fig. 4. Block diagram representation of two-mass suspension

As an example, the two-mass vertical model shown in Fig. 3 can either be represented in block diagram form as shown by Fig. 4, or by the following equations:

$$
\begin{gathered}
m \ddot{z}+c\left(\dot{z}-\dot{z}^{\prime}\right)+k\left(z-z^{\prime}\right)=f_{a} \\
m^{\prime} \ddot{z}^{\prime}+c\left(\dot{z}^{\prime}-\dot{z}\right)+c^{\prime}\left(\dot{z}^{\prime}-\dot{z}_{t}\right)+k\left(z^{\prime}-z\right)+k^{\prime}\left(z^{\prime}-z_{t}\right)=-f_{a}
\end{gathered}
$$

which can in turn be written using a matrix equation 


$$
\begin{gathered}
M \underline{\ddot{z}}+C \underline{\dot{z}}+K \underline{z}=C_{t} \dot{z}_{t}+K_{t} z_{t}+B_{a} f_{a} \\
\underline{z}=\left[z z^{\prime}\right]^{T} \\
M=\left[\begin{array}{cc}
m & 0 \\
0 & m^{\prime}
\end{array}\right] ; \quad C=\left[\begin{array}{cc}
c & -c \\
-c\left(c+c^{\prime}\right)
\end{array}\right] ; \quad K=\left[\begin{array}{cc}
k & -k \\
-k\left(k+k^{\prime}\right)
\end{array}\right]
\end{gathered}
$$

This can subsequently be converted into state-space form as follows:

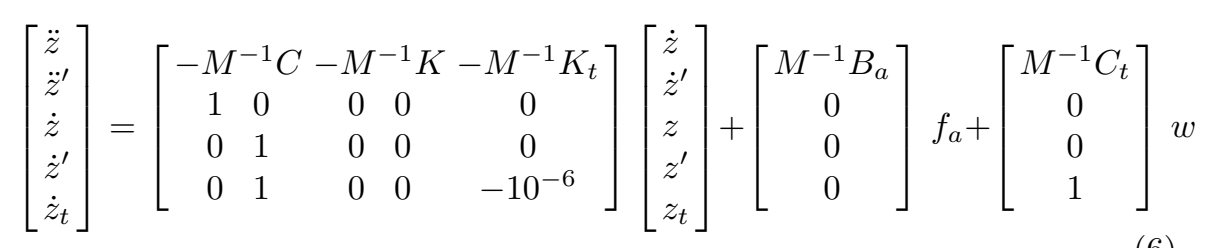

As observed in the previous subsection, there is a design trade-off between the suspension deflection and the ride quality, the latter being quantified by a frequency-weighted r.m.s. acceleration, and so it is primarily these two quantities that are required as outputs. Accordingly the corresponding output equation to give the secondary suspension deflection and the acceleration of the secondary mass is

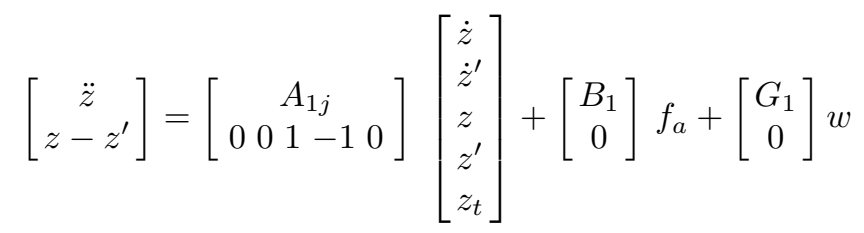

Here the track input is a scalar, i.e. $\underline{w}=\left[\dot{z}_{t}\right]$, and the actuator (control) input is also a scalar $\underline{u}=\left[f_{a}\right]$, but in general it is obvious that this kind of representation can be extended to accommodate multiple inputs.

The example doesn't include the more complicated modelling of the wheelset dynamics [2], i.e. it doesn't include creep forces etc., but the general principle can readily include these aspects.

\subsection{Control Concepts}

Active control can be applied to both secondary and primary suspensions. The control can be directed towards the response to the deterministic (intended) inputs such as curves or gradients, or towards the response to irregularities, or to a combination of the two. Although in principle this offers a very broad range of possibilities, in practice it's possible to provide more focussed categorisation of the major opportunities as follows:

- Secondary suspension control

(a) Tilting trains - enables higher speeds through curves via improved response to deterministic track features. 
(b) Active secondary suspensions - provides better ride quality, i.e. an improved response to track irregularities.

- Primary suspension (control of wheels and wheelsets)

(a) Active steering - gives improved curving leading to reduced wheel and rail wear (improved response to deterministic track features).

(b) Active stability/guidance - provides improved stability and/or higher speed (improved response to track irregularities).

In practice tilting and active secondary suspensions are sufficiently distinct in an operational sense that separate descriptions are appropriate, whereas the technology required to achieve the two primary suspension options is very similar and so they are described together, even though the control strategies will be very different.

\section{Tilting trains}

The basic idea is to lean the vehicles inwards on curves to reduce the acceleration felt by the passengers [3]. However this acceleration as a vehicle passes onto a railway curve does not rise suddenly: there is a transition from the straight to the curve, usually lasting around 2 seconds, which is a deliberate design feature so that passengers are not made uncomfortable by too sudden an application of sideways acceleration. Normally the track is leaned inward or "canted" to reduce the lateral acceleration experienced by the passengers, and this also increases steadily through the transition - Fig. 5.

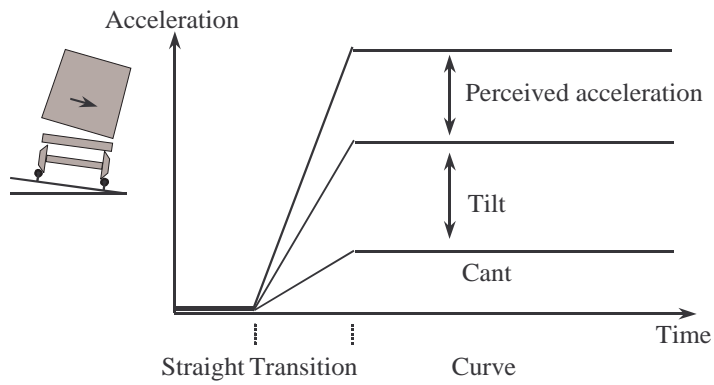

Fig. 5. Tilting concept

At higher speeds the curving acceleration rises, and the transition will also be more severe because the duration of the transition will reduce, that is unless the track is changed. This is where tilt comes in, to bring the acceleration back to the level it was before. However, it is not only what happens in the steady curve that is important, but also the dynamic response during the transition. 
Ideally the tilt angle of the body should rise progressively, perfectly aligned both with the onset of curving acceleration and the rising cant angle.

Some means of providing tilt of the vehicle body relative to the bogie is achieved using a combination of a tilting mechanism and a set of actuators. Essentially this achieves active control of the secondary roll suspension, but for tilting the aim is only to respond to curve inputs so as to reduce the lateral acceleration perceived by the passengers; the response to lateral track irregularities is largely unaffected and characterised by the components in the secondary suspension. To avoid motion sickness it is now normal not to compensate fully for the curving acceleration, with typically around 60-70\% compensation being used. It is particularly important to respond quickly in curve transitions, i.e. as well as providing appropriate steady-state curving performance, and achieving this without degrading the straight track ride quality is a non-trivial issue. Control can either use a feed-forward system where the track features are determined and used to provide an appropriate command input for the tilt actuation system, or a feedback approach in which lateral accelerations on the vehicle body or bogie are measured, or a combination of the two.

\section{Active secondary suspensions}

The essential theoretical concepts involved were identified back in the 1970s, and are largely based upon the use of so-called "skyhook" damping in which the actuators are made to appear like "virtual dampers" connected to an absolute datum (see Fig. 6), which enables significantly higher levels of damping to be introduced into the suspension dynamic modes without compromising the performance at higher frequencies [4]. Active control also means that characteristics of the different modes (vertical, pitch, lateral, yaw, roll) can be influenced in a much more flexible manner, for example the rotational modes can be made significantly softer than the translational modes, which further improves the suspension performance. The improvements can be used either to provide a better ride quality, or to achieve higher speed of operation, or to enable the use of a lower track quality.

\section{Active primary suspensions}

Before discussing the use of active elements, it's necessary to understand how the conventional railway wheelset works. It consists of two coned or otherwise profiled wheels rigidly connected by an axle. On straight track the wheelset runs in a centralised position, but when a curve is encountered the wheelset naturally moves outwards; this causes the outer wheel to run on a larger radius and the inner on a smaller radius. Being connected by the axle the wheels must still rotate at the same rotational speed, so the outer wheel moves faster along the track, and the effect is to make the wheelset go around the curve, i.e. a natural mechanical steering action. 


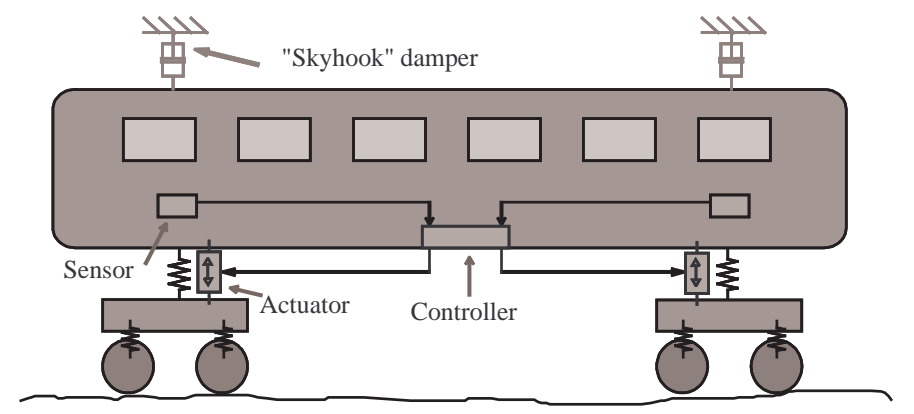

Fig. 6. Active secondary suspension concept

However there is a problem, which arises when the dynamics of the wheelset are assessed. The combination of the profiled wheels and the creep forces mentioned previously is to create an oscillatory system, a combined lateral and yaw motion known as "hunting". Adding mechanical dampers doesn't provide stability, and the normal solution is to have two wheelsets connected to a bogie frame by means of longitudinal springs. These springs create non-conservative creep forces at the wheel-rail contact point and stabilise the hunting motion.

However, on a curve these stabilising longitudinal springs produce forces which interfere with the natural curving action of the wheelset. The result is that on the tighter curves the wheel flange will be in contact with the side of the rail, causing wear of the wheels, wear of the rails and often significant amounts of noise. There is therefore a difficult design trade-off: stiff springs give stable high-speed running, but poor curving; soft springs mean that the curving performance is better, but stable running is only possible at low speeds.

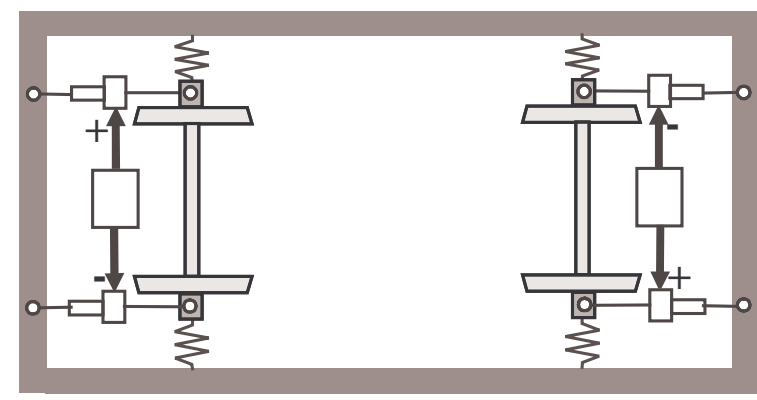

Fig. 7. Active primary suspension concept

The variety of possibilities for controlling the wheels and wheelsets is very large, and based upon an analysis of possible configurations (mechanical 
scheme, axle type, control objective and actuation technology) [5]. However the most basic scheme is shown in Fig. 7, in which longitudinal actuators between the bogie frame and the ends of the wheelsets are controlled in a differential sense to apply a yaw torque to each wheelset. This torque can either be used to provide a steering action on curves, or it can be used to provide stability to the wheelset without affecting the natural curving, or a combination of the two.

\section{Case Study: Control of Secondary Suspensions - Tilting Trains}

The concept of tilting trains and associated general control concepts have already been discussed in section 1.4. In this case study we will encounter specific issues on the modelling, control objectives, assessment and control design of a tilting train.

\subsection{Historical facts on tilt control}

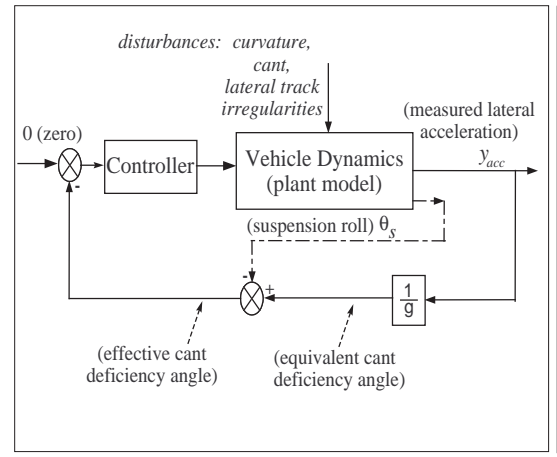

(a) Intuitive partial nulling control (early-type)

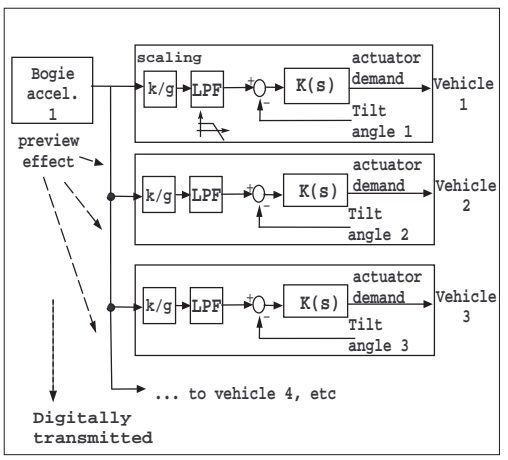

(b) Command driven with precedence control (commercial)

Fig. 8. Tilt control schemes

Early tilt control systems were based solely upon local-per vehicle measurements (Fig.8a), however it proved impossible at the time to get an appropriate combination of straight track and curve transition performance. Interactions between suspension and controller dynamics (with the sensors being within the control loop) led to control limitations and stability problems. Since then, tilt controllers have evolved in an incremental sense, the end result of which is a control structure which is not optimised from a system point of view. The 
industrial norm nowadays is to utilise precedence control devised in the early 1980s as part of the UK's Advanced Passenger Train development [4]. In this scheme a bogie-mounted accelerometer from the vehicle in front is used to provide "precedence" (à priori track information) (via appropriate inter-vehicle cable/signalling connections), carefully designed so that the delay introduced by the filter compensates for the preview time corresponding to approximately a vehicle length (Fig.8b). Normally nowadays a single command signal would be generated from the first vehicle and transmitted digitally with appropriate time delays down the train. Consequently the velocity and the direction of travel are important factors for the correct operation of the tilt system.

The command-driven with precedence strategy proved to be successful and it is nowadays used by most tilting train manufacturers. However it is a complex scheme, direction-sensitive, signal connections between trains are required, while the tilt system performance can be optimised for a specific route operation. Moreover, leading vehicles have inferior performance due to lack of precedence.

Nevertheless achieving a satisfactory local tilt control strategy remains an important issue because of the system simplifications and more straightforward failure detection.

\subsection{Tilting Vehicle Modelling}

The modelling of the baseline railway vehicle is based upon a linearised endview model version (Fig.9). It includes the lateral and roll dynamics of both the body and the bogie plus a state from the airspring dynamics, resulting to a $9^{\text {th }}$ order model.

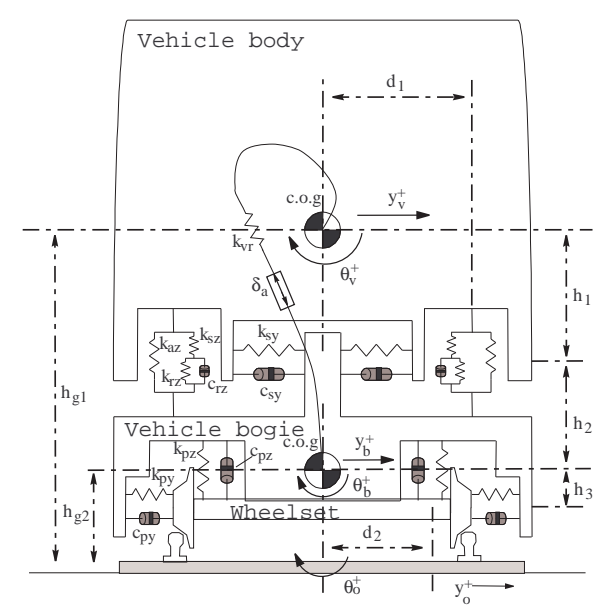

(a) End-view model

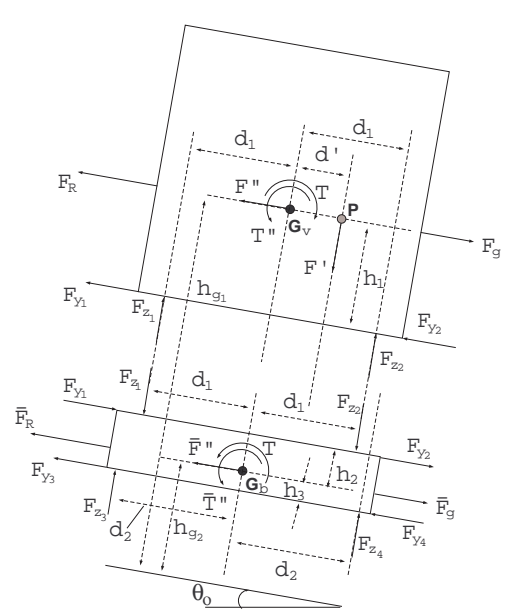

(b) Free body diagram

Fig. 9. Tilting vehicle end-view diagram with actuation system 
A pair of linear airsprings represents the vertical suspensions, which only contribute to the roll motion of the vehicle (the vertical degrees of freedom are ignored). The model also contains the stiffness of an anti-roll bar connected between the body and the bogie frame. Detailed wheelset dynamics were not included for simplicity.

To provide active tilt a rotational displacement ideal actuator, is included in series with the roll stiffness ('active anti-roll bar (ARB)' [6]). The ARBsystem is assumed to provide up to a maximum tilt angle of 10 degrees. The advantages of active ARBs results from their relative simplicity, i.e. small weight increase, low cost, easily fitted as an optional extra to build or as a retro-fit.

The mathematical models of increasing complexity, via Newton's laws, were developed to encapsulate the lateral and roll dynamics of the tilting vehicle system. The equations of motion are given below with all variables and parameter values provided in Appendix A. For the vehicle body:

$$
\begin{array}{r}
m_{\mathrm{v}} \ddot{y}_{\mathrm{v}}=-2 k_{\mathrm{sy}}\left(y_{\mathrm{v}}-h_{1} \theta_{\mathrm{v}}-y_{\mathrm{b}}-h_{2} \theta_{\mathrm{b}}\right)-2 c_{\mathrm{sy}}\left(\dot{y}_{\mathrm{v}}-h_{1} \dot{\theta}_{\mathrm{v}}-\dot{y}_{\mathrm{b}}-h_{2} \dot{\theta}_{\mathrm{b}}\right) \\
-\frac{m_{\mathrm{v}} v^{2}}{R}+m_{\mathrm{v}} g \theta_{\mathrm{o}}-h_{\mathrm{g} 1} m_{\mathrm{v}} \ddot{\theta}_{\mathrm{o}} \\
i_{\mathrm{vr}} \ddot{\theta}_{\mathrm{v}}=-k_{\mathrm{vr}}\left(\theta_{\mathrm{v}}-\theta_{\mathrm{b}}-\delta_{\mathrm{a}}\right)+2 h_{1}\left\{k_{\mathrm{sy}}\left(y_{\mathrm{v}}-h_{1} \theta_{\mathrm{v}}-y_{\mathrm{b}}-h_{2} \theta_{\mathrm{b}}\right)\right. \\
\left.+c_{\mathrm{sy}}\left(\dot{y}_{\mathrm{v}}-h_{1} \dot{\theta}_{\mathrm{v}}-\dot{y}_{\mathrm{b}}-h_{2} \dot{\theta}_{\mathrm{b}}\right)\right\} \\
+m_{\mathrm{v}} g\left(y_{\mathrm{v}}-y_{\mathrm{b}}\right)+2 d_{1}\left\{-k_{\mathrm{az}}\left(d_{1} \theta_{\mathrm{v}}-d_{1} \theta_{\mathrm{b}}\right)\right. \\
\left.-k_{\mathrm{sz}}\left(d_{1} \theta_{\mathrm{v}}-d_{1} \theta_{\mathrm{r}}\right)\right\}-i_{\mathrm{vr}} \ddot{\theta}_{\mathrm{o}}
\end{array}
$$

For the vehicle bogie:

$$
\begin{array}{r}
m_{\mathrm{b}} \ddot{y}_{\mathrm{b}}=2 k_{\mathrm{sy}}\left(y_{\mathrm{v}}-h_{1} \theta_{\mathrm{v}}-y_{\mathrm{b}}-h_{2} \theta_{\mathrm{b}}\right)+2 c_{\mathrm{sy}}\left(\dot{y}_{\mathrm{v}}-h_{1} \dot{\theta}_{\mathrm{v}}-\dot{y}_{\mathrm{b}}-h_{2} \dot{\theta}_{\mathrm{b}}\right) \\
-2 k_{\mathrm{py}}\left(y_{\mathrm{b}}-h_{3} \theta_{\mathrm{b}}-y_{\mathrm{o}}\right)-2 c_{\mathrm{py}}\left(\dot{y}_{\mathrm{b}}-h_{3} \dot{\theta}_{\mathrm{b}}-\dot{y}_{\mathrm{o}}\right) \\
-\frac{m_{\mathrm{b}} v^{2}}{R}+m_{\mathrm{b}} g \theta_{\mathrm{o}}-h_{\mathrm{g} 2} m_{\mathrm{b}} \ddot{\theta}_{\mathrm{o}} \\
i_{\mathrm{br}} \ddot{\theta}_{\mathrm{b}}=k_{\mathrm{vr}}\left(\theta_{\mathrm{v}}-\theta_{\mathrm{b}}-\delta_{\mathrm{a}}\right)+2 h_{2}\left\{k_{\mathrm{sy}}\left(y_{\mathrm{v}}-h_{1} \theta_{\mathrm{v}}-y_{\mathrm{b}}-h_{2} \theta_{\mathrm{b}}\right)\right. \\
\left.+c_{\mathrm{sy}}\left(\dot{y}_{\mathrm{v}}-h_{1} \dot{\theta}_{\mathrm{v}}-\dot{y}_{\mathrm{b}}-h_{2} \dot{\theta}_{\mathrm{b}}\right)\right\} \\
-2 d_{1}\left\{-k_{\mathrm{az}}\left(d_{1} \theta_{\mathrm{v}}-d_{1} \theta_{\mathrm{b}}\right)-k_{\mathrm{sz}}\left(d_{1} \theta_{\mathrm{v}}-d_{1} \theta_{\mathrm{r}}\right)\right\} \\
+2 d_{2}\left(-d_{2} k_{\mathrm{pz}} \theta_{\mathrm{b}}-d_{2} c_{\mathrm{pz}} \dot{\theta}_{\mathrm{b}}\right) \\
+2 h_{3}\left\{k_{\mathrm{py}}\left(y_{\mathrm{b}}-h_{3} \theta_{\mathrm{b}}-y_{\mathrm{o}}\right)+c_{\mathrm{py}}\left(\dot{y}_{\mathrm{b}}-h_{3} \dot{\theta}_{\mathrm{b}}-\dot{y}_{\mathrm{o}}\right)\right\}-i_{\mathrm{br}} \ddot{\theta}_{\mathrm{o}}
\end{array}
$$

for the (additional) airspring state:

$$
\dot{\theta}_{\mathrm{r}}=-\frac{\left(k_{\mathrm{sz}}+k_{\mathrm{rz}}\right)}{c_{\mathrm{rz}}} \theta_{\mathrm{r}}+\frac{k_{\mathrm{sz}}}{c_{\mathrm{rz}}} \theta_{\mathrm{v}}+\frac{k_{\mathrm{rz}}}{c_{\mathrm{rz}}} \theta_{\mathrm{b}}+\dot{\theta}_{\mathrm{b}}
$$


Local track references were used, and both the translation and rotation of these reference axes associated with curves were allowed for in the equations $\left(F^{\prime \prime}, \bar{F}^{\prime \prime}\right.$ and $T^{\prime \prime}, \bar{T}^{\prime \prime}$. Moreover, (9) includes an end moment effect, $F^{\prime}=m_{\mathrm{v}} g\left(y_{\mathrm{v}}-y_{\mathrm{b}}\right)$, modelling the roll effect of the body weight due to the lateral displacement of its centre of gravity on the curve. However, this effect was neglected in the case of the bogie mass owing to the high stiffness of the primary suspensions. The complexity of the system is clearly shown by the set of equations of motion.

Note also that substantial coupling exists between the lateral and roll motions which result in two sway modes combining both lateral and roll movement, and their centres located at points other than the vehicle centre of gravity. An 'upper sway' mode, its node appears above the body c.o.g., with predominantly roll movement; and a 'lower sway' mode, its node located below the body c.o.g., characterised predominantly by a lateral motion. The modal analysis of the vehicle is shown in Table 1 , with the modes being close to the industrial-norm.

Table 1. ARB vehicle model dynamic modes

\begin{tabular}{|c|c|c|}
\hline Mode & Damping (\%) & Frequency $(\mathrm{Hz})$ \\
\hline Body lower sway & 16.5 & 0.67 \\
\hline Body upper sway & 27.2 & 1.50 \\
\hline Bogie lateral & 12.4 & 26.80 \\
\hline Bogie roll & 20.8 & 11.10 \\
\hline Airspring mode & 100.0 & 3.70 \\
\hline
\end{tabular}

For system analysis and control design, the system is written in the state space form

$$
\begin{array}{r}
\dot{x}=A x+B u+B_{w} w \\
y=C x+H w
\end{array}
$$

where,

$$
\begin{aligned}
& x=\left[\begin{array}{lllllllll}
y_{\mathrm{v}} & \theta_{\mathrm{v}} & y_{\mathrm{b}} & \theta_{\mathrm{b}} & \dot{y}_{\mathrm{v}} & \dot{\theta}_{\mathrm{v}} & \dot{y}_{\mathrm{b}} & \dot{\theta}_{\mathrm{b}} & \theta_{\mathrm{r}}
\end{array}\right]^{T}, u=\left[\delta_{a}\right], \ldots \\
& w=\left[R^{-1} \theta_{o} \dot{\theta}_{o} \ddot{\theta}_{o} y_{o} \dot{y}_{o}\right]^{T}
\end{aligned}
$$

For simulation purposes only, disturbance signals $\theta_{o}, \dot{\theta}_{o}, y_{o}$ should be incorporated into the $A$ matrix (in this case the stochastic track includes the filtering effects of the wheelset). It should be noted, that the necessary $C$ and $D$ output matrices, for control design, can be formed from the relevant rows (depending on the required outputs) of the given $A$ and $B$ matrices. A number of outputs is available such as displacements, velocities, accelerations of 
the vehicle body and bogies and also displacements, velocities of the active elements.

A series of transient tests ensure that the vehicle behaves in a similar manner to its full scale equivalent (real) vehicle (for the passive model, with actuator inactive). The track profiles, both deterministic and stochastic, used for this purpose can be seen in Section 2.3.
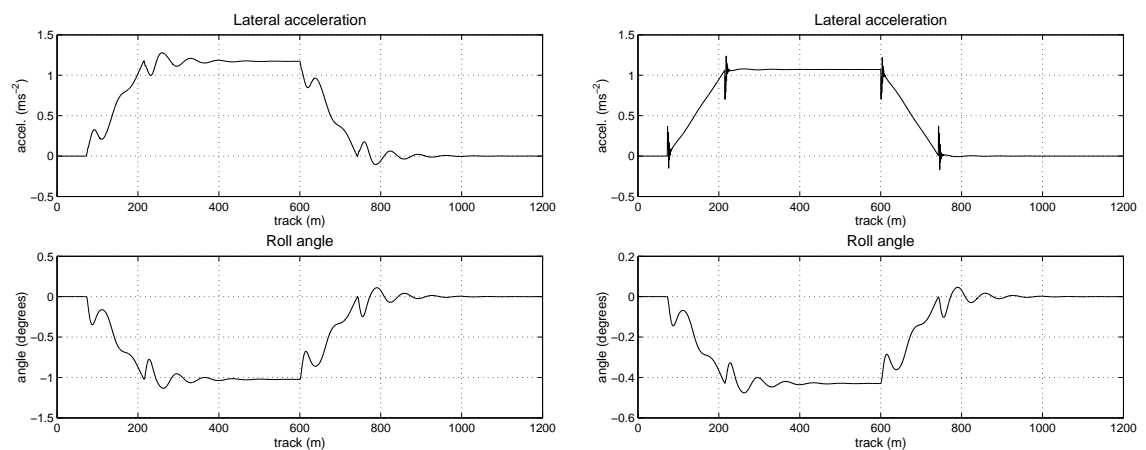

(a) Vehicle body

(b) Vehicle bogie
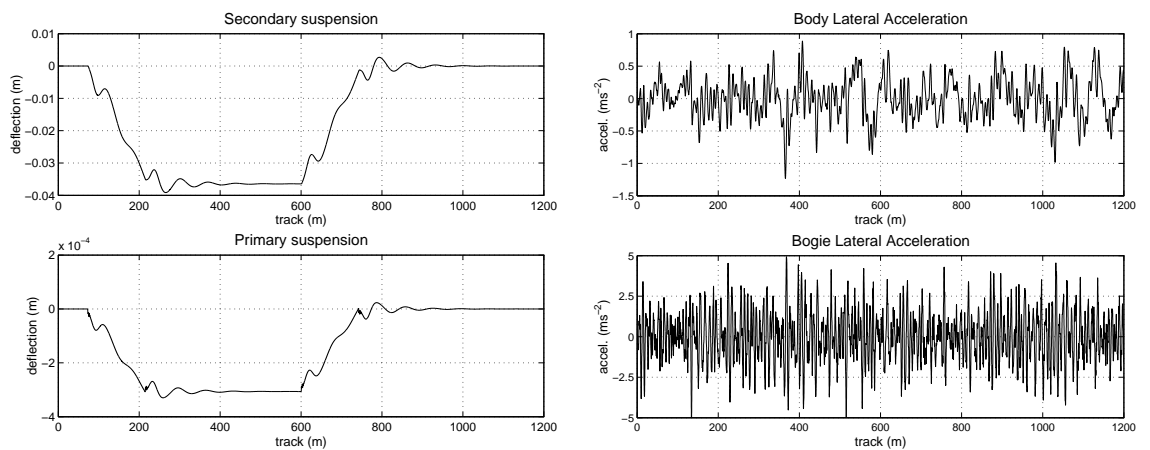

(c) Suspension deflections on det. track

(d) Lateral acceleration on stochastic track@ $45(\mathrm{~m} / \mathrm{s})$

Fig. 10. Vehicle body/bogie time histories @ $45(\mathrm{~m} / \mathrm{s})$

In this passive (non-tilting) case, a nominal vehicle speed of $v_{o}=45 \frac{\mathrm{m}}{\mathrm{s}}$ $\left(162 \frac{\mathrm{km}}{\mathrm{h}}\right)$ is assumed, and the designed cant deficiency at this speed is $\frac{v_{o}^{2}}{R}-$ $g \theta_{o}=5.83^{\circ}$ or $1.0\left(\frac{m}{s^{2}}\right)$. Fig. 10(a) shows the lateral acceleration and corresponding roll angle for the body mass. The lateral acceleration level is what the passengers would experience on the curve transition, and it is provided by a lateral accelerometer placed on the vehicle body c.o.g. The peak value is $13.0 \% \mathrm{~g}$, while the steady-state value is around $11.93 \% \mathrm{~g}$ at a forward vehicle speed of $45(\mathrm{~m} / \mathrm{s})$. The increase in lateral acceleration is because the lateral 
suspension acts significantly lower than the body centre of gravity, and as a consequence the body roll-outwards on curves (steady-state $1.0^{\circ}$ ).

In the case of the bogie mass, Fig. 10(b), the roll-out is less (steady state value of $0.43^{\circ}$ ) due to the stiffer primary suspensions. The steady-state level of the bogie lateral acceleration of $10.7 \% \mathrm{~g}$ is closer to the cant deficiency for which the track was designed by the civil engineers. Note that more high frequency components are now present due to the harsh environment of the bogie system.

Fig. 10(c) shows a comparison between the lateral displacements of the vehicle body and bogie. The effect of the two different sets of suspensions is clearly evident. The soft secondary suspensions cause a displacement of $36.5(\mathrm{~mm})$ in steady-state, while the primary suspensions owing to the high stiffness have hardly been displaced by $0.3(\mathrm{~mm})$ in steady-state.

It is also important to test the behaviour of the vehicle model on the straight track irregularities, which are the primary cause of ride quality degradation. Fig. 10(d) presents the time histories for the lateral acceleration of both the body and the bogie travelling on straight track. The nominal vehicle speed is assumed $45(\mathrm{~m} / \mathrm{s})$. The bogie due to its harsh environment has an R.M.S. value of lateral acceleration equal to $16.4 \% \mathrm{~g}$, while the soft secondary suspensions filter out a large amount of high frequencies and leave the body with an R.M.S. lateral acceleration of $2.932 \% \mathrm{~g}$.

\subsection{Tilt Control Requirements and Assessment Approach}

\section{Requirements}

The performance of tilt control systems on the curve transitions is critical; most importantly the passenger ride comfort provided by the tilting vehicle should not be (significantly) degraded compared to the non-tilting vehicle speeds. The main objectives of any tilt control system are:

1. to provide an acceptably fast response to changes in track cant and curvature (deterministic track features)

2. not to react significantly to track irregularities (stochastic track features)

However, any tilt control system directly controls the secondary suspension roll angle (i.e. the inclination of the body) and not the vehicle lateral acceleration. Hence, there is a fundamental trade-off between the vehicle curve transition response and straight track performance. Moreover, for reasons of human perception, designers utilise partial tilt compensation. In such a case the passenger will still experience a small amount of acceleration on steady curve, in order to minimise motion sickness phenomena.

From a control design point of view the objectives of the tilt system can be translated (in terms of shaping the loop) as: increasing the response of the system at low frequencies (deterministic track features), while reducing the 
high frequency system response (stochastic track features) and maintaining stability.

Note that in this study the tilt controllers should provide $60 \%$ tilt compensation on steady curve at high speed (for reasons of passenger motion sickness as discussed earlier in the chapter), i.e. the curving acceleration at the increased speed is reduced to $40 \%$ of the non-tilting case (at the same speed) rather than to zero. Although this strictly is known as partial-nulling tilt, for simplicity we will refer to it as nulling tilt.

\section{Tilt Control Assessment}

As mentioned previously, the performance of the tilt controller on the curve transition is critical, and their assessment is based upon a combination of two approaches, i.e. the ' $P_{C T}$ factors' and the 'ideal tilting' assessment.

The former is based upon a comprehensive experimental/empirical study which provides the percentage of (both standing and seated) passengers who feel uncomfortable during the curve transition. The latter method emphasises the assessment of the control system performance by determining the deviations from the concept of "ideal tilting", i.e. the tilt action follows the specified tilt compensation in an ideal manner, defined on the basis of the maximum tilt angle and cant deficiency compensation factor. This combination of parameters is optimised via the $P_{C T}$ factors approach to choose a basic operating condition. The procedure follows a minimisation approach of dynamic effects relative to tilt angles, roll velocities and lateral accelerations (Fig.11); more details can be found in [7].

- $\left|\ddot{y}_{m}-\ddot{y}_{m_{i}}\right|$, the deviation of the actual lateral acceleration $\ddot{y}_{m}$ from the ideal lateral acceleration $\ddot{y}_{m_{i}}$, in the time interval between 1 s before the start of the curve transition and $3.6 \mathrm{~s}$ after the end of the transition.

- $\left|\dot{\theta}_{m}-\dot{\theta}_{m_{i}}\right|$, the deviation of the actual absolute roll velocity $\ddot{\theta}_{m}$ from the ideal absolute roll velocity $\ddot{\theta}_{m_{i}}$, in the time interval between 1 s before the start of the curve transition and 3.6s after the end of the transition.

Regarding the straight track case the 'rule-of-thumb' currently followed by designers is to allow a lateral ride quality degradation of the tilting train by no more than a specified margin of between $7.5 \%-10 \%$ compared with the non-tilting vehicle.

\section{Track inputs}

The deterministic track input employed for simulation purposes consists of a curved section with a radius of $1000 \mathrm{~m}$ superimposed by a maximum track cant angle of $155 \mathrm{~mm}$ (i.e. $6^{\circ}$ ), with a tilting speed of $209 \mathrm{~km} / \mathrm{h}$ over a track length of $1200 \mathrm{~m}$. The stochastic track inputs represent the irregularities in the track alignment on both straight track and curves, and these were characterised by an approximate spatial spectrum equal to $\frac{\Omega_{l} v^{2}}{f_{\mathrm{s}}^{3}}\left(\frac{\mathrm{m}^{2}}{\text { cycle } / m}\right)$ with a lateral track roughness $\left\{\Omega_{l}\right\}$ of $0.33 \cdot 10^{-8} \mathrm{~m}[12]$. 


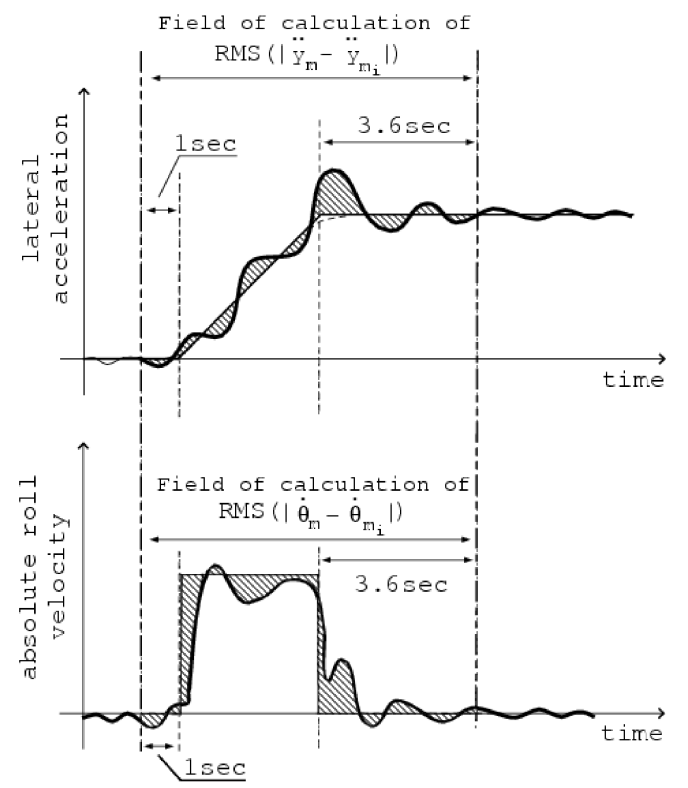

Fig. 11. "Ideal Tilting"- Calculation of deviation of actual from ideal responses for acceleration and roll velocity[7]

\subsection{Conventional Tilt Control}

This section deals with the early-type conventional nulling control scheme and the current practice followed by industry i.e. command-driven with precedence approach.

\section{Classical nulling control strategy}

In this intuitively formulated control strategy (as seen from Figure 8a), which is a classical application of SISO feedback control, the signal from a bodymounted lateral accelerometer provides a measurement of the curving acceleration experienced by the passengers. The controller would drive the feedback signal to zero and therefore give $100 \%$ compensation, so a portion of the suspension roll angle (actual tilt angle) is included in the feedback path, chosen to provide the required $60 \%$ compensation.

The control input comprises an angular displacement $\left(\delta_{\mathrm{a}}\right)$ provided by a rotary actuator in series with the anti-roll bar, which in turn provides a torque to the vehicle body. Note that the reference signal is 'zero', i.e. the system is subject to track disturbances only. The feedback signal for partial tilt compensation is the effective cant deficiency angle $\theta_{d m}^{\prime}$ which is given by

$$
\theta_{d m}^{\prime}=\left(-\lambda_{1} \frac{\ddot{y}_{\mathrm{vm}}}{g}-\lambda_{2} \theta_{2 s r}\right)
$$


where $\ddot{y}_{\mathrm{vm}}$ is the lateral acceleration felt by the passengers as measured from an accelerometer on the body c.o.g (18), and $\theta_{2 s r}$ is the secondary suspension roll angle (19).

$$
\begin{aligned}
& \ddot{y}_{\mathrm{vm}}=\frac{v^{2}}{R}-g\left(\theta_{o}+\theta_{\mathrm{v}}\right)+\ddot{y}_{\mathrm{v}} \\
& \theta_{2 s r}=\theta_{\mathrm{v}}-\theta_{\mathrm{b}}
\end{aligned}
$$

Note the effect of the deterministic track included in (18). Factors $\lambda_{1}, \lambda_{2}$ ensure partial tilt and for $60 \%$ compensation need to be set to 0.615 and 0.385 respectively, taking in account bogie roll-out in (19).

Remark: The sign of the feedback signal is inverted for correct application of negative feedback based upon the chosen axes system. All lateral motions $(y)$ are positive inwards to the curve center and all roll motions $(\theta)$ are positive clockwise. However, the lateral accelerometer (a mass on a spring) measures positive acceleration outwards (18). Hence, the acceleration is translated into a positive cant deficiency angle ${ }^{1}$, combined with a portion of the suspension roll, and is then fed back (note that $\lambda_{1} \frac{\ddot{y}_{v m}}{g} \geq \lambda_{2} \theta_{2 s r}$ ). The combined signal will thus be a positive (w.r.t. the choice of directions) angle, which if fed back using negative feedback will cause the controller to provide a negative tilt angle (i.e. anti-clockwise rotation), consequently destabilising the system. Thus inversion of the sign of the feedback signal is necessary to guarantee proper body rotation for correct compensation.

Conclusions about the stability of the closed-loop may be drawn by investigating the open-loop frequency response of the system. The nominal open-loop frequency responses from $u:=\delta_{a}$ to $y_{1}:=\theta_{d m}^{\prime}$, and $y_{2}:=\theta_{2 s r}$ can be seen in Fig. 12. Note that, while gain reduction is required to stabilise the closed-loop system, the opposite applies in the case of fast tilt response. Hence, there must be a compromise between the tilt response and the ride quality.

A closer investigation of the open-loop poles and zeros of the transfer function $G_{y_{1} u}(s)=\frac{\theta_{d m}^{\prime}(s)}{\delta_{a}(s)}$ reveals that, while the system is open-loop stable, is also non-minimum phase due to the existence of two RHP zeros at $(s-29.4)$ and $(s-6.02)$, Table 2 . The pole-zero map of the uncompensated system $G_{y_{1} u}(s)$ can be seen in Fig. 13. The presence of RHP-zeros imposes a fundamental limitation on control, and high controller gains induce closed-loop instability.

Integral action is necessary to guarantee zero effective cant deficiency angle on steady curve. We design a classical PI controller $K_{p i}(s)=k_{g}\left(1+\frac{1}{s \tau_{i}}\right)$, with the proportional term limiting the extra phase-lag at increasing frequency. The settings for $k_{g}$ and $\tau_{i}$ were adjusted to suffice both the deterministic and stochastic criteria, i.e. $k_{g}=0.225, \tau_{i}=0.4 s$ (these can be slightly

\footnotetext{
${ }^{1}$ Cant deficiency defines the difference between the existing degree of cant and the degree required to fully eliminate the effect of centrifugal force at maximum allowable speed
} 


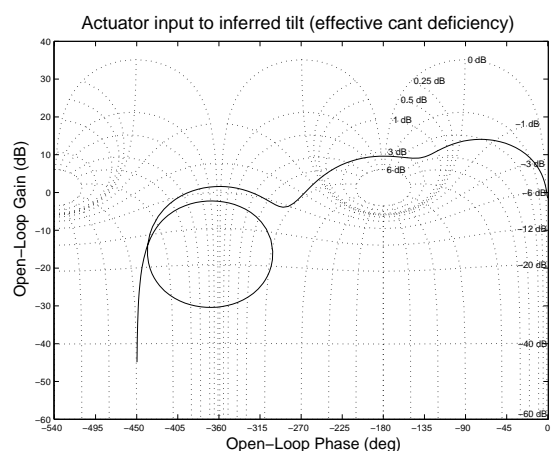

(a) Tilt model open-loop responses (Nichols)

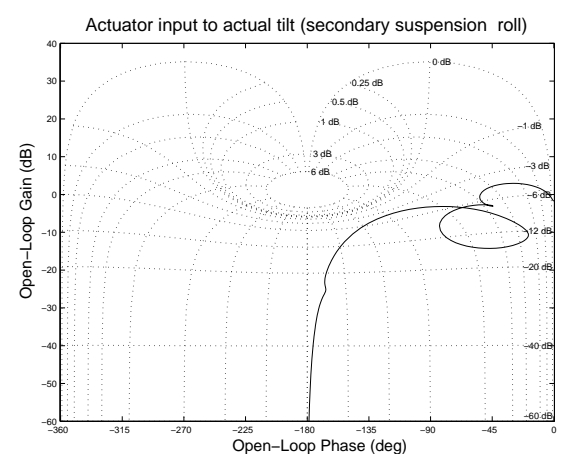

(b) Effective cant deficiency open-loop

Fig. 12. Tilt angle open-loop

Table 2. Open-loop poles and zeros of $G_{y_{1} u}(j \omega)$

\begin{tabular}{rr}
\hline \multicolumn{1}{c}{ OL poles } & \multicolumn{1}{c}{ OL zeros } \\
\hline$-20.87 \pm 167.34 \mathrm{j}$ & $-2.41 \pm 125.94 \mathrm{j}$ \\
$-14.57 \pm 68.38 \mathrm{j}$ & $\mathbf{2 9 . 3 6} \pm \mathbf{0 . 0 0 j}$ \\
$-2.56 \pm 9.03 \mathrm{j}$ & $-40.73 \pm 0.00 \mathrm{j}$ \\
$-0.69 \pm 4.12 \mathrm{j}$ & $-26.18 \pm 0.00 \mathrm{j}$ \\
$-23.22 \pm 0.00 \mathrm{j}$ & $\mathbf{6 . 0 2} \pm \mathbf{0 . 0 0 j}$ \\
- & $-3.83 \pm 3.13 \mathrm{j}$ \\
\hline
\end{tabular}

further tuned via optimization). Table 3 presents the overall controller assess-

Pole-Zero map of $G_{y_{1} u}(j \omega)$

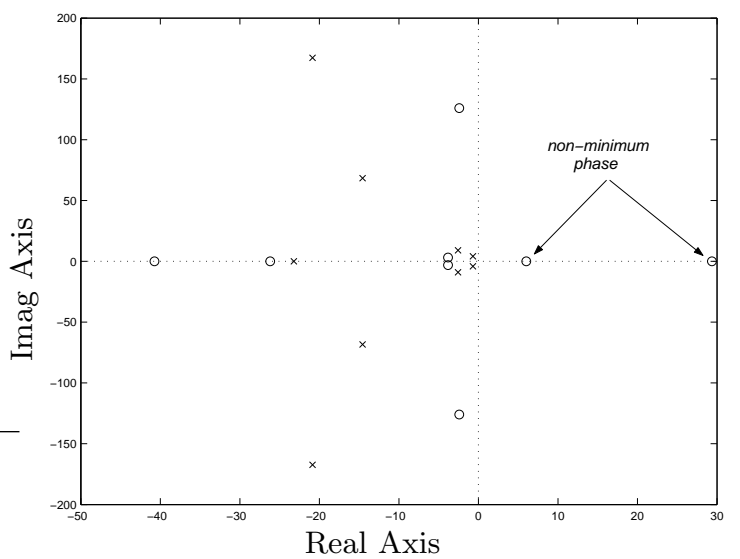

Fig. 13. Pole-zero map of uncompensated OL 
Table 3. Early-type nulling PI control assessment @ 58(m/s)

\begin{tabular}{llc}
\hline \multicolumn{3}{c}{ DETERMINISTIC } \\
\hline Lateral accel. & - steady-state & n/a $(\% \mathrm{~g})$ \\
(actual vs ideal) & - R.M.S. deviation error & $5.555(\% \mathrm{~g})$ \\
& - peak value & $19.510(\% \mathrm{~g})$ \\
Roll gyroscope & - R.M.S. deviation & $0.032(\mathrm{rad} / \mathrm{s})$ \\
& - peak value & $0.086(\mathrm{rad} / \mathrm{s})$ \\
(PCT-factor) & - peak jerk level & $10.286(\% \mathrm{~g} / \mathrm{s})$ \\
& - standing & $71.411(\% \mathrm{of}$ passng) \\
& - seated & $22.640(\% \mathrm{of}$ passng) \\
\hline \multicolumn{2}{c}{ STOCHASTIC } \\
\hline Passenger comfort - R.M.S. passive (equiv.) & $3.778(\% \mathrm{~g})$ \\
& - R.M.S. active & $3.998(\% \mathrm{~g})$ \\
& - degradation & $5.802(\%)$ \\
\hline
\end{tabular}

ment, while Fig. 14 and Fig. 15 present the simulation results on curved track and the corresponding frequency responses respectively. Clearly the response is very slow with the steady-state values for acceleration, roll angle and roll rate profiles not met. Note that, due to the vehicle body inertia at the start and the end of curve, the body roll angle initially has an inverse response and then rises slowly up to the required steady-state value. The difference between the control input $\delta_{a}$ and the body roll $\theta_{\mathrm{v}}$ is due to the torque imposed by the secondary suspension subject to the curving forces on the vehicle body. Thus, additional control effort is demanded to overcome this extra torque and regulate the body roll (via the suspension roll portion) to the desired value. There is also great difficulty in controlling the roll rates appropriately, which has a detrimental effect on the overall system performance. Note that the secondary suspension deflection is given by $x_{2 d f l}=\left\{y_{\mathrm{v}}-h_{1} \theta_{\mathrm{v}}-\left(y_{\mathrm{b}}+h_{2} \theta_{\mathrm{b}}\right)\right\}$.

Moreover, the complementary sensitivity $T$, in Fig. 15(b), shows that the control action in the frequency range $\left[0.4\left(\frac{\mathrm{rads}}{\mathrm{s}}\right), 4\left(\frac{\mathrm{rads}}{\mathrm{s}}\right)\right]$ does not affect the system, which causes a slow response (i.e. insufficient bandwidth). Moreover, higher frequency components enter owing to the sway mode resonances in the interval $\left[4\left(\frac{\mathrm{rads}}{\mathrm{s}}\right), 10\left(\frac{\mathrm{rads}}{\mathrm{s}}\right)\right]$, with the control action incapable of improving the performance due to $|S|>1$. The uncompensated and compensated openloop responses are presented in Fig. 15(a), with the latter emphasising the OL integral action at low frequencies.

The difficulties in the early-type classical nulling strategy are the following: (i) the sensor exists within the control loop resulting in interactions between controller and suspension dynamics, and (ii) the system is also non-minimum phase and there is a stability threshold point set by the non-minimum phase zero, which result in major limitations in the controller design.

Later on in the section we will see how we can improve the performance of nulling-type schemes with the use of modern control methods. 


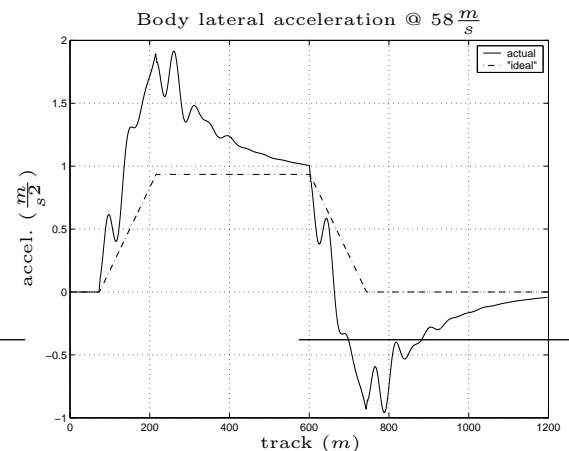

(a) Passenger acceleration

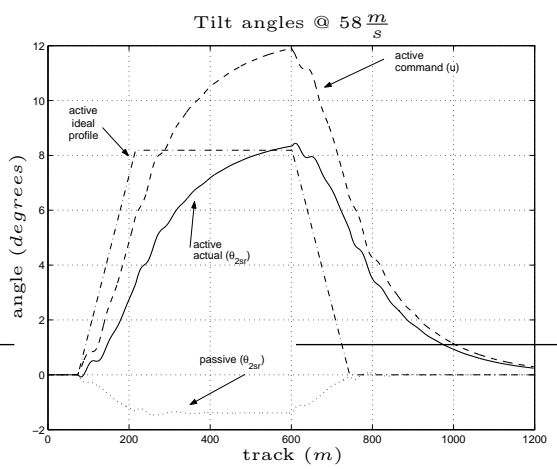

(c) Tilt angles

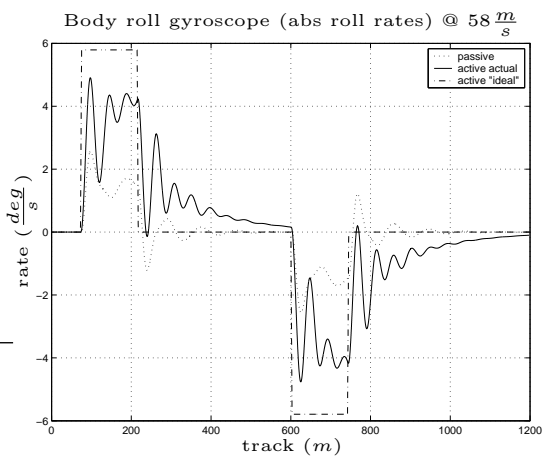

(b) Roll rate

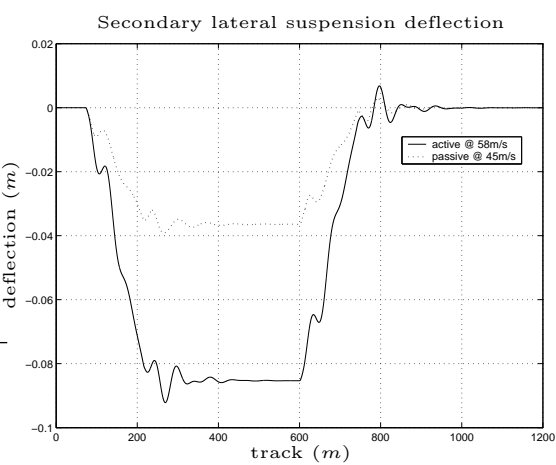

(d) Suspension Deflection

Fig. 14. Early-type nulling PI scheme for deterministic track

\section{Command-driven with precedence control}

The problems with the early-type nulling schemes and also with heavy low pass filtering in attempting local command-driven strategies (to reduce the effect of bogie high frequency components in the feedback signal used), led to the currently used by industry command-driven with precedence scheme (the concept can be seen in Fig. 8(b)).

For illustrative purposes we consider the command-driven with precedence scheme in Fig. 16. It employs an accelerometer on the leading bogie of the leading vehicle (a preview of $29 \mathrm{~m}$ was assumed) provided the curving acceleration signal, passed via a $0.45 \mathrm{~Hz}$ low pass filter. The signal is then processed to provide $60 \%$ compensation for the lateral acceleration ( $K$ is set to 0.6 ). The actuator is controlled using an additional feedback of tilt angle (i.e. the suspension roll).

For appropriate comparison to the local tilt strategies, the filter delay was chosen to match the precedence time, however this can be changed to emphasise precedence information if necessary. The transfer function of the 


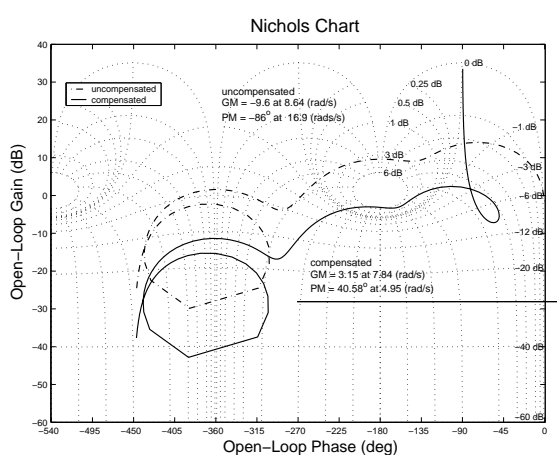

(a) Uncompensated and compensated $\mathrm{OL}$

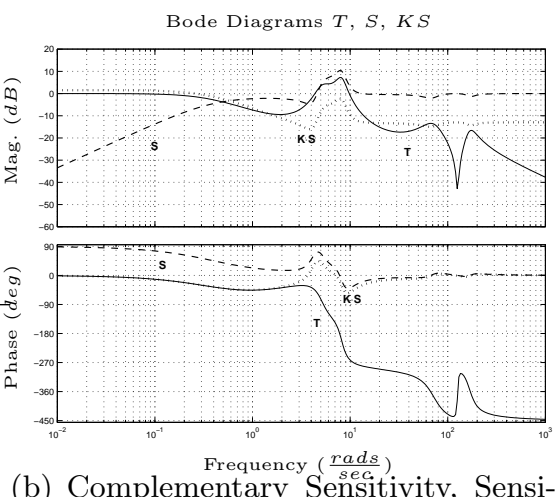

tivity and Control Sensitivity

Fig. 15. Early-type nulling PI system frequency responses

LP filter is given by

$$
H_{L P 2}(s)=\frac{w_{c_{2}}^{2}}{s^{2}+2 \zeta_{2} w_{c_{2}}+w_{c_{2}}^{2}}, \quad w_{c_{2}}=2 \pi \times 0.45, \zeta_{2}=0.707
$$

and the time delay introduced is $t_{d_{L P}}=\frac{2 \zeta_{2} w_{c_{2}}}{w_{c_{2}}^{2}}$, which for the current case is $0.5 s$. Thus for the precedence to match the filter delay, it takes $l=58\left(\frac{\mathrm{m}}{\mathrm{s}}\right) \times$ $0.5 s=29 m$ precedence, i.e. approximately 1.5 vehicle length. Note that the tilting response for the leading vehicle will unavoidably be too late.

The leading vehicle controller was chosen as $K_{p i}(s)=\left(1+\frac{1}{s 0.5}\right)$. For the trailing vehicle, the controller designed to actively tilt the body is a PI compensator in series with a low-pass filter $(\mathrm{LPF})^{2}$, in order to remove high frequencies from the secondary suspension roll (those are introduced due to the bogie roll contribution). The overall controller transfer function is

$$
K_{\text {total }}(s)=\left(\frac{1.5+s 0.75}{s 0.5}\right) \times \frac{400}{s^{2}+28.28 s+400}
$$

The compensated and uncompensated open loop together with the overall compensator frequency response can be viewed in Fig. 17(a). The corresponding sensitivity and complementary sensitivity of the closed loop system are presented in Fig. 17(b), where it is evident that the control action influences the system over a wider range of frequencies compared to the classical nulling case.

A set of time-domain results for the deterministic track case is shown in Fig. 18, where it is obvious that the precedence scheme is superior to the classical nulling approach. The tilt controller performance is presented in

\footnotetext{
2 the LP filter will be redundant in the case of actuator dynamics, i.e. actuator will have limited bandwidth by default.
} 

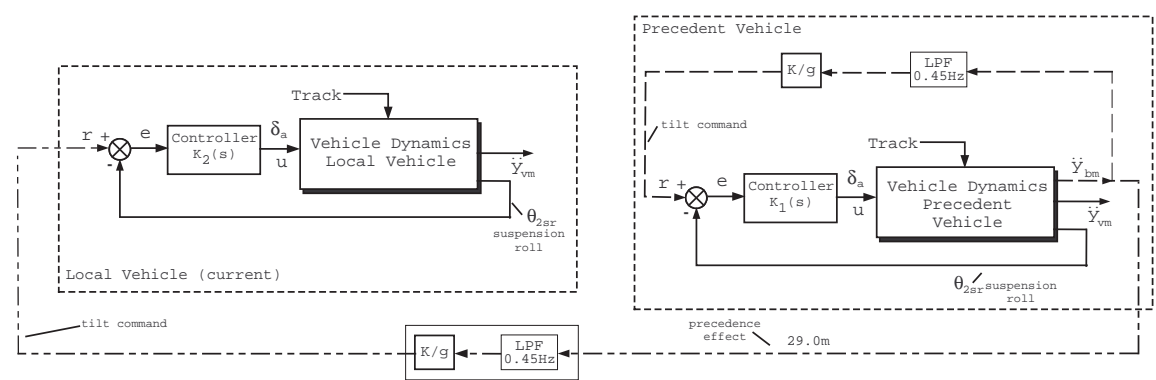

(a) Block Diagram

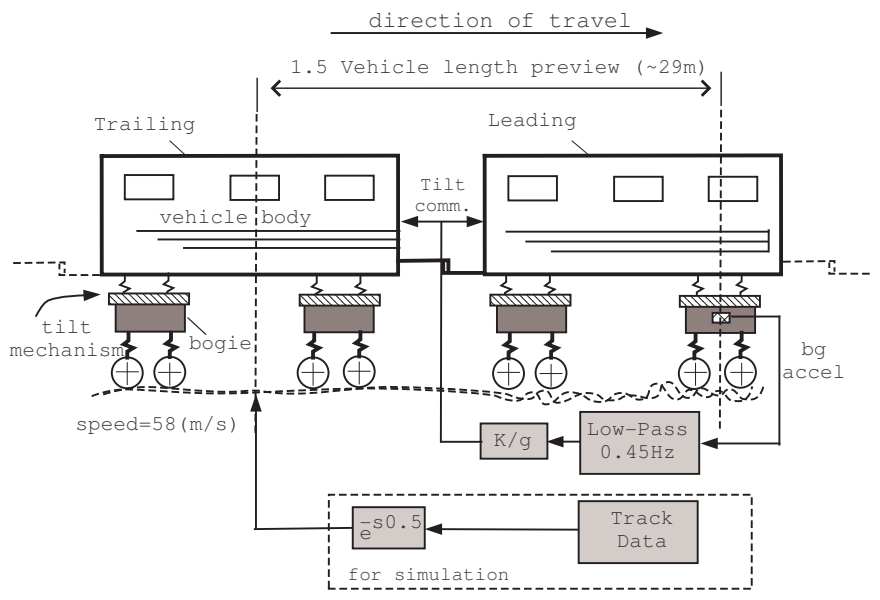

(b) Interpretation for simulation purposes

Fig. 16. Command-driven with precedence approach

Table 4, and it is closer to the ideal performance as expected in all cases. In the stochastic case there is an improvement in ride quality by the active system, because the precedence time matches the filter delay meaning that the reference and track input are uncorrelated, thus the tilt command will compensate for long wavelengths.

Emphasising more precedence information improves the deterministic performance, subject of course to the amount of precedence used, i.e. too much precedence (over-precedence) can be disastrous for the normal operation of the train (tilt action will apply on straight track segments much sooner than the intended start of the curve!). In addition, the amount of precedence used will influence the stochastic ride quality either in a positive or negative way depending upon the correlation of the signals (in the case where the precedence time differs from the filter delay, the reference and track input signals are no longer uncorrelated). It should be noted that, even in the precedence 


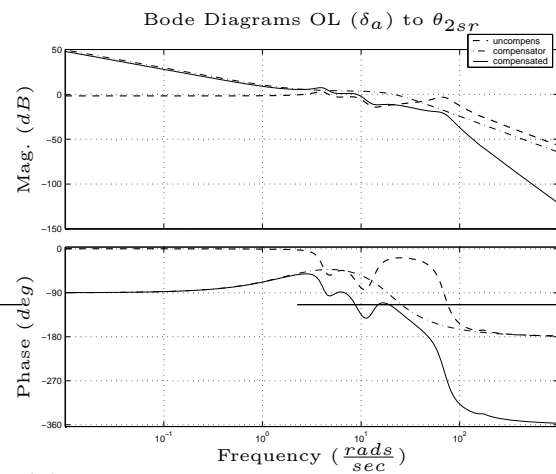

(a) Compensated and uncompensated $\mathrm{OL}$
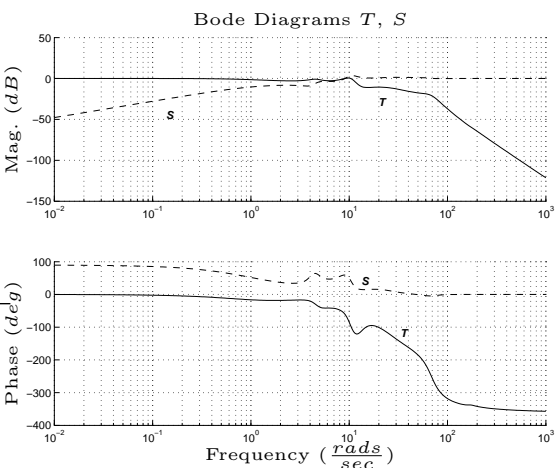

(b) Complementary Sensitivity, Sensitivity

Fig. 17. Command-driven with precedence system frequency responses

Table 4. Command-driven with precedence - assessment @ 58(m/s)

\begin{tabular}{llc}
\hline \multicolumn{3}{c}{ DETERMinistic } \\
\hline Lateral accel. & - steady-state & $9.53(\% \mathrm{~g})$ \\
(actual vs ideal) & - R.M.S. deviation error & $1.54(\% \mathrm{~g})$ \\
& - peak value & $12.18(\% \mathrm{~g})$ \\
Roll gyroscope & - R.M.S. deviation & $0.018(\mathrm{rad} / \mathrm{s})$ \\
& - peak value & $0.104(\mathrm{rad} / \mathrm{s})$ \\
(P-factor) & - peak jerk level & $6.80(\% \mathrm{~g} / \mathrm{s})$ \\
& - standing & $47.62(\% \mathrm{of}$ passng) \\
& - seated & $13.455(\%$ of passng) \\
\hline \multicolumn{3}{c}{ STOCHASTIC } \\
\hline Passenger comfort & - R.M.S. passive (equiv.) & $3.78(\% \mathrm{~g})$ \\
& - R.M.S. active & $3.31(\% \mathrm{~g})$ \\
& - degradation & $-12.12(\%)$ \\
\hline
\end{tabular}

schemes, sensors located on each vehicle (i.e. local sensors) are used to ensure the correct operation of the overall tilting system (the sensors are always present for safety purposes).

\subsection{Nulling-type tilt via robust control techniques}

In this section we will see how to improve the performance nulling-type schemes by employing robust control methods, in order to achieve comparable results to the command-driven with precedence scheme. In particular the case study involves an LQG/LTR approach [11] and a multiple objective $H_{\infty} / H_{2}$ scheme [12]. 


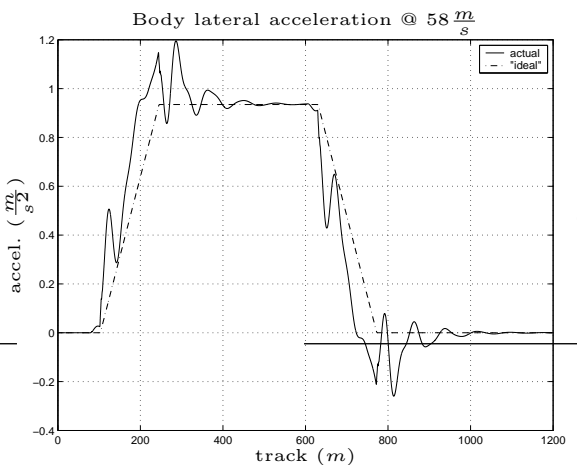

(a) Passenger acceleration

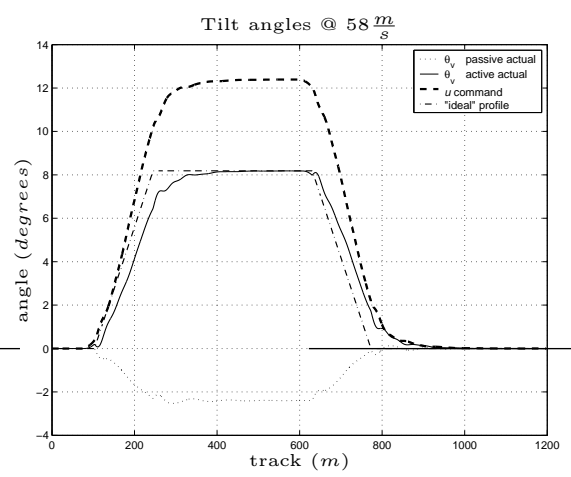

(c) Tilt angles

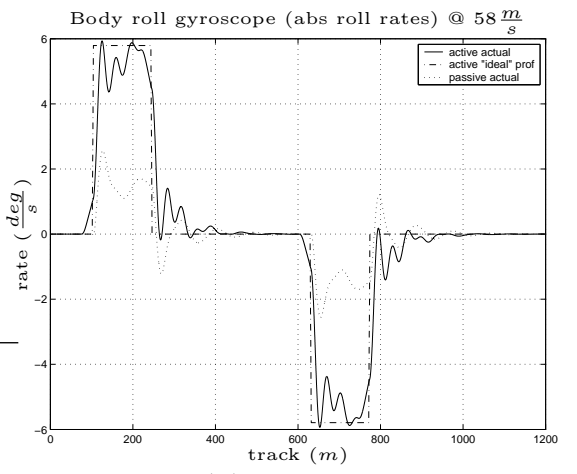

(b) Roll rate

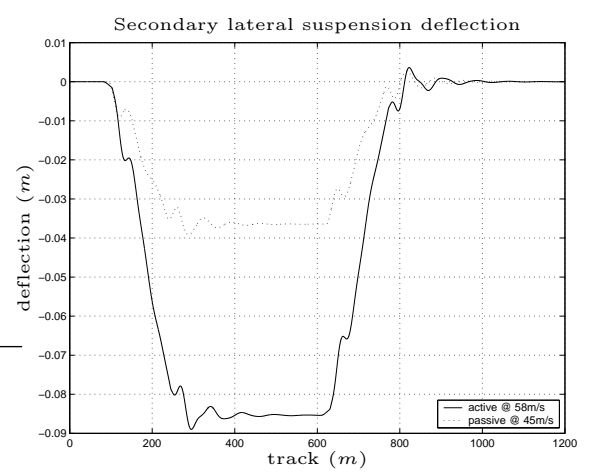

(d) Suspension Deflection

Fig. 18. Command-driven with precedence on deterministic track

\section{LQG/LTR nulling-type tilt control}

Linear Quadratic Gaussian control is well documented in [8],[10], defining the following state-space plant model

$$
\begin{aligned}
& \dot{x}=A x+B u+\Gamma w \\
& y=C x+v,
\end{aligned}
$$

where $w, v$ are (ideally) white uncorrelated process and measurement noises which excite the system, and are characterised by covariance matrices $W, V$ respectively. The separation principle can be applied to first find the optimal control $u=-K_{r} x$ which minimises (23)

$$
J=\lim _{T \rightarrow \infty} \frac{1}{T} E\left\{\int_{0}^{T}\left[x^{T} Q x+u^{T} R u\right] d \tau\right\}
$$

where $K_{r}=R^{-1} B^{T} X$ and $X$ is the positive semi-definite solution of the following Algebraic Riccati Equation (ARE) 


$$
[X-I]\left[\begin{array}{cc}
A-B R^{-1} B^{T} \\
-Q & -A^{T}
\end{array}\right]\left[\begin{array}{c}
I \\
X
\end{array}\right]=0
$$

Next find the optimal state estimate $\hat{x}$ of $x$ where

$$
\hat{x}=A \hat{x}+B u+K_{f}(y-C \hat{x})
$$

to minimise $E\left\{[x-\hat{x}]^{T}[x-\hat{x}]\right\}$. The optimal Kalman gain is given by $K_{f}=$ $Y C^{T} V^{-1}$ and $Y$ is the positive semi-definite solution of the following ARE

$$
\left[\begin{array}{ll}
Y & -I
\end{array}\right]\left[\begin{array}{cc}
A^{T} & -C^{T} V^{-1} C \\
-\Gamma W \Gamma^{T} & -A
\end{array}\right]\left[\begin{array}{c}
I \\
Y
\end{array}\right]=0
$$

Weighting matrices $Q$ (pos. semidefn.), $R$ (pos. defn.) for control, and $W$ (pos. semidefn.), $V$ (pos. defn.) for estimation, can be tuned to provide the desired result. Note that it is also possible to follow the dual procedure, i.e. solve for the state estimate sub-problem and next for the optimal gain sub-problem (although this is not considered in our study).

For synthesizing the tilt controller we consider a simple extension to the classical nulling approach in an optimal control framework by deriving the SISO model, from $\delta_{\mathrm{a}}$ to $\theta_{d m}^{\prime}$, with all disturbance signals set to zero. Thus, on measurement is effectively use for the Kalman filter.

We will synthesize the controller using the weighting matrices $Q, R, W, V$ purely as tuning parameters until an appropriate design is obtained. In particular, the structure of the LQG tilt compensator is found by shaping the principal gains of the system, i.e. return ratios. First the LQR is synthesized via $Q, R$ to obtain a satisfactory a satisfactory return ratio $-K_{r}(s I-A)^{-1} B$, with the Kalman Filter designed via $W, V$ such that the return ratio at the input of the compensated plant converges sufficiently close to $-K_{r}(s I-A)^{-1} B$ over the frequency range of interest (Loop Transfer Recovery to recover as much as possible of the robust properties of LQR).

For disturbance rejection and/or reference tracking (which is zero in this case), the system should be augmented using an extra state, the integral of the effective cant deficiency $\theta_{d m}^{\prime}$. This approach will produce an optimal controller with integral action [8]. Hence, the system is becomes

$$
\left(\begin{array}{c}
\dot{x} \\
\dot{x}^{\prime}
\end{array}\right)=\left(\begin{array}{cc}
A & 0 \\
C^{\prime} & 0
\end{array}\right)\left(\begin{array}{c}
x \\
x^{\prime}
\end{array}\right)+\left(\begin{array}{c}
B \\
0
\end{array}\right) u
$$

where $x^{\prime}=\int \theta_{d m}^{\prime}$ and $C^{\prime}$ is the selector matrix for integral action and is found from $\theta_{d m}^{\prime}=C^{\prime} x$. The control signal has the form

$$
u=-\left(K_{p} K_{i}\right)\left(\begin{array}{c}
x \\
x^{\prime}
\end{array}\right)
$$

We start with the simplest possible choices for $Q, R$ 


$$
Q=\left(\begin{array}{cc}
0_{9 \times 9} & 0_{9 \times 1} \\
0_{1 \times 9} & q_{i}
\end{array}\right), \quad R=1
$$

thus adjusting only the weight of the integral state, and imposing no constraints on the remaining states. Fig.19 illustrates the return ratio $-K_{r}(s I-A)^{-1} B$ for various $q_{i}$. We choose the return ratio for $q_{i}=100$ with a crossover of approx 20rad/s, to recover for in the next steps. However a simple calculation of the transmission zeros for the design plant reveals a non-minimum phase zeros at approximately $6.0 \mathrm{rad} / \mathrm{s}$ (this is characteristic for such a setup in tilting trains [12]), thus making full recovery cumbersome. For illustration, the usual LTR procedure is followed up to the limit of recovery allowed from the non-minimum phase zero (usually the achievable bandwidth of the system is less than half of the RHP zero frequency [10]).

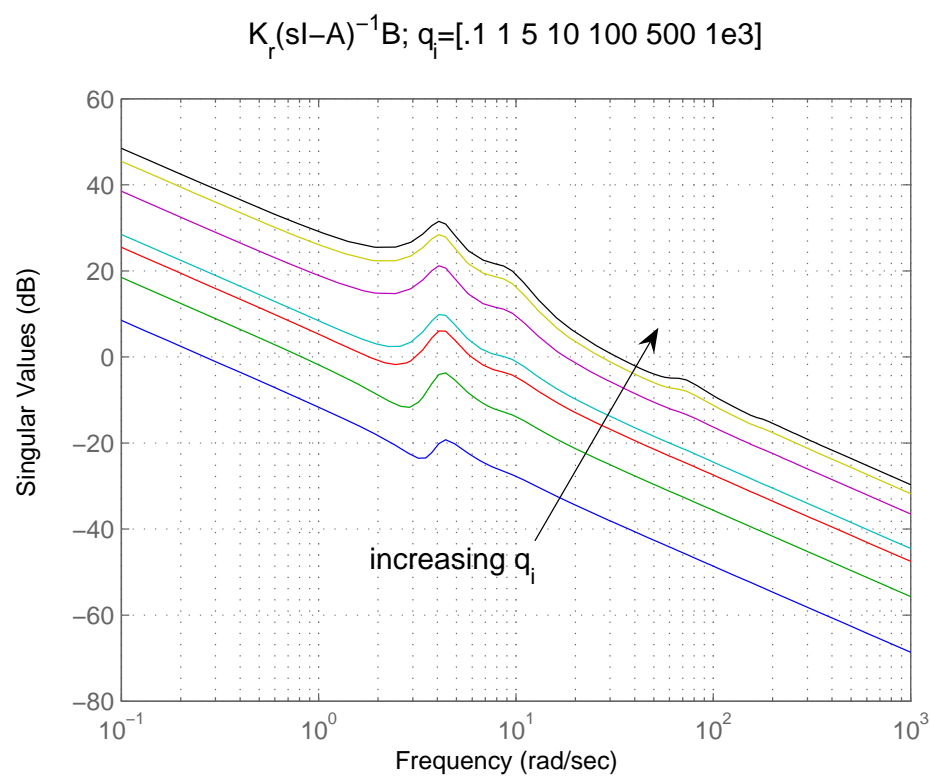

Fig. 19. Return ration $-K_{r}(s I-A)^{-1} B$ for various $q_{i}$

Moreover, for the design of the Kalman Filter, the zero eigenvalue of the augmented $A$ matrix needs to be placed just to the left of the origin for the solution to exist. For controller implementation this should move back to the origin for proper integration. equal to $B, 1$ respectively (still for the SISO model). Setting $\Gamma=B$ refers to any (virtual) disturbances on the plant acting via the input, rather than the actual track disturbances from the track.

The sensor noise covariance matrices is set to $V=1$ (can be reduced to characterise better quality measurements), while the process noise covariance matrix is set to $W=W_{o}+\tilde{w} I\left(W_{o}=0\right)$. Fig.20 illustrates the amount of 
recovery at plant output for increasing values of $\tilde{w}$. It is seen that there is no point in recovering after $\tilde{w}=500$ as appropriate integral action is already recovered and the actual crossover limit is placed by the non-minimum phase zero.

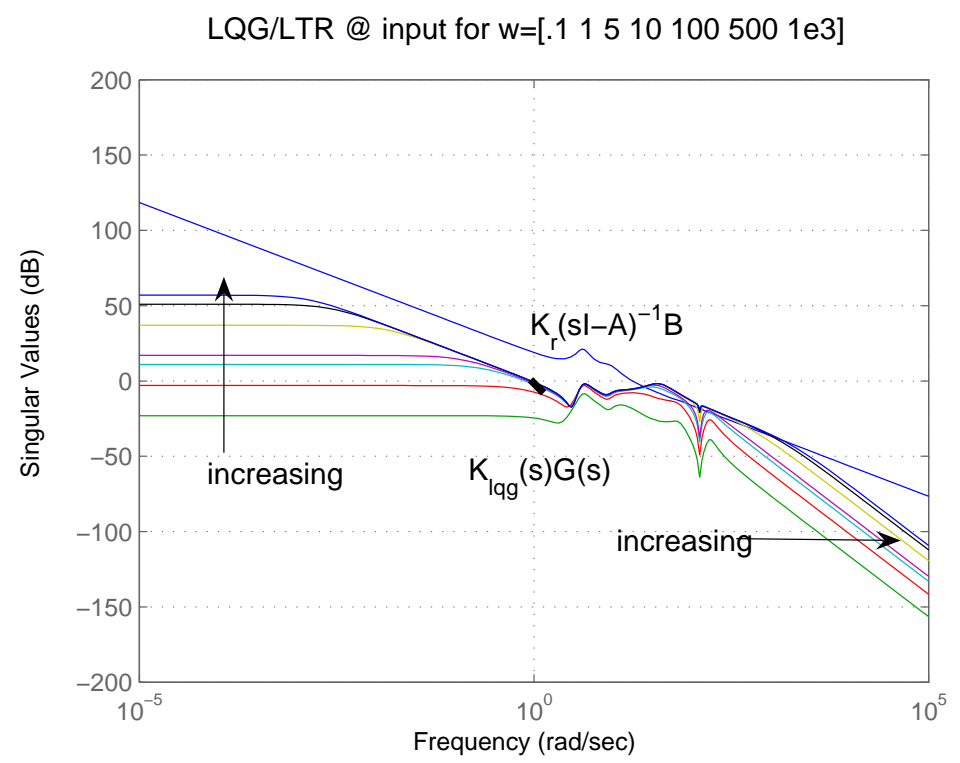

Fig. 20. LTR at plant output for increasing $\tilde{w}$

Fig. 21 presents the principal gains of the designed closed loop system w.r.t. sensitivity $S$ and complementary sensitivity $T$. The bandwidth of the system is rather low (approx. 1rad/s due to the NMP zero) however there is some compensation at higher frequencies which cater for few stochastic components (on straight track ride).

The synthesized LQG controller realization is given by

$$
K_{l q g} \stackrel{s}{=}\left[\begin{array}{c|c}
A-B K_{r}-K_{f} C & K_{f} \\
\hline-K_{r} & 0
\end{array}\right]
$$

and is $10^{\text {th }}$ order and its frequency response can be seen in Fig. 22. This can be further reduced either in an open loop or closed sense [10], however at the expense of performance quality.

The time domain results for the lateral acceleration felt by the passengers and the related body tilt angle can be seen in Fig. 23, while the performance assessment of the controller is presented in Table 5. It is seen that although the LQG-based is a simple straightforward optimal extension of the classical 


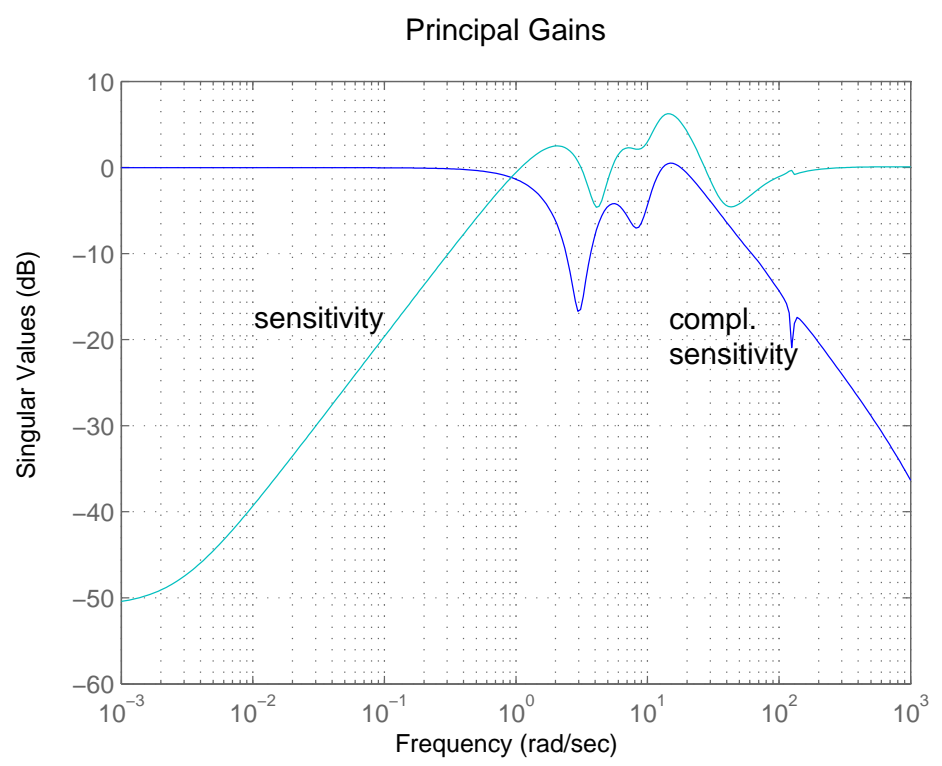

Fig. 21. Designed system Sensitivity and Compl. Sensitivity with LQG controller

Table 5. LQG nulling-type control assessment @ 58(m/s)

\begin{tabular}{llc}
\hline \multicolumn{3}{c}{ DETERMINISTIC } \\
\hline Lateral accel. & - steady-state & $9.53(\% \mathrm{~g})$ \\
(actual vs ideal) & - R.M.S. deviation error & $4.09(\% \mathrm{~g})$ \\
& - peak value & $17.3(\% \mathrm{~g})$ \\
Roll gyroscope & - R.M.S. deviation & $0.033(\mathrm{rad} / \mathrm{s})$ \\
& - peak value & $0.101(\mathrm{rad} / \mathrm{s})$ \\
(PCT-factor) & - peak jerk level & $8.98(\% \mathrm{~g} / \mathrm{s})$ \\
& - standing & $65.76(\%$ of passng) \\
& - seated & $19.95(\%$ of passng) \\
\hline \multicolumn{3}{c}{ STOCHASTIC } \\
\hline Passenger comfort - R.M.S. passive (equiv.) & $3.78(\% \mathrm{~g})$ \\
& - R.M.S. active & $3.97(\% \mathrm{~g})$ \\
& - degradation & $4.83(\%)$ \\
\hline
\end{tabular}

nulling scheme, the performance is much improved (emphasizing robustness with the additional damping injected).

It is worth mentioning that the performance of the controller can be further improved by using extra sensor information, i.e. passenger acceleration, body roll rate, vehicle yaw rate [13]. The design system in this case will be nonsquare with more outputs than inputs, as a result the LQG controller will be non-square (more inputs than outputs). However, we can still synthesize via the separation principle, but make the system square for LTR [8]. 


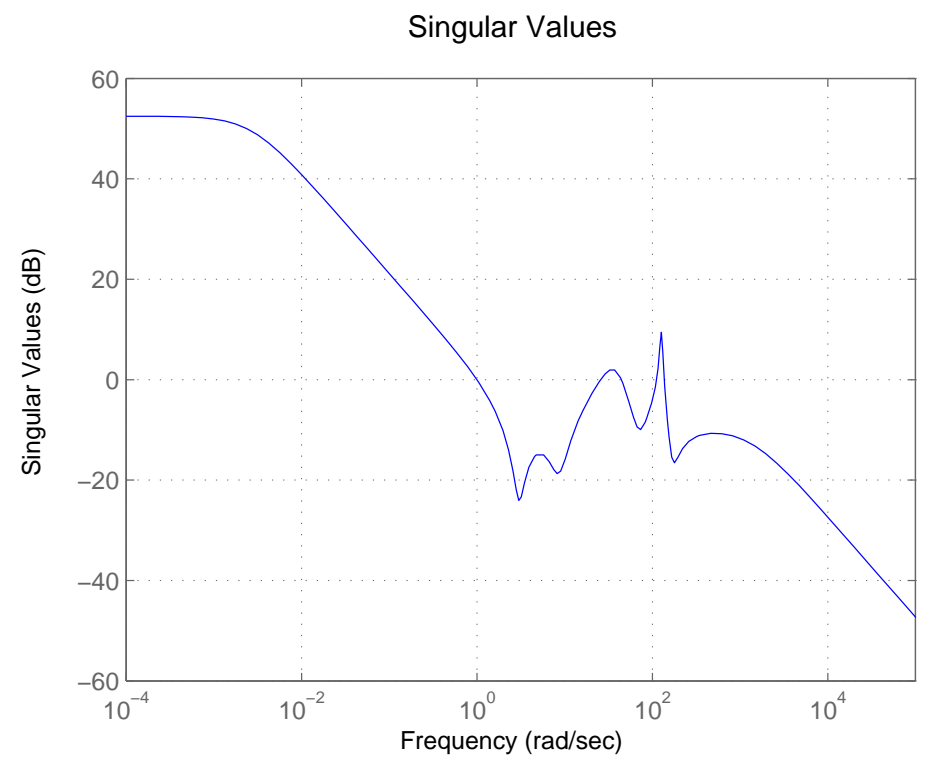

Fig. 22. LQG controller principal gain

\subsection{Multi-objective $\mathrm{H}_{\infty} / \mathrm{H}_{2}$ nulling-type control via LMIs}

It is well known that the $H_{\infty}$ norm of a system represents the worst-case energy transfer between (bounded energy) disturbances to (bounded-energy) regulated outputs, and as a result can be conservative when disturbances are naturally modelled as persistent or white noise signals. In cases where the interests falls upon minimising the RMS value of a regulated output, the $H_{2}$ norm [10] of the corresponding closed-loop transfer function is a more appropriate measure of stochastic performance. This section considers a multipleobjective $H_{\infty} / H_{2}$ via LMIs [14] design method for the local nulling-type tilt control [15]. Details on some preliminary concepts related to the following design procedure can be found in Appendix B.

The design objectives are formulated as an optimisation problem, defined in the generalised-regulator setting shown in Fig. 24, where $P(S)$ and $K(s)$ are the generalised plant (inclusive of all weighting factors) and the controller to be designed. The vector of external disturbances was set to $w=\left[w_{1} w_{2}\right]^{T}$, where $w_{1}$ denotes $\left(\frac{1}{R}\right)$ the (deterministic) track-curvature (low-frequency) disturbance signal and $w_{2}$ is the (stochastic) lateral track position (higherfrequency) signal $y_{o}$. Scaling factors $W_{i_{1}}, W_{i_{2}}$ emphasise the relative weighting between the two disturbances for the design. Note that in this case we choose to employ two outputs $y_{1}$ and $y_{2}$ which are the measured body lateral acceleration and the secondary suspension roll angle, respectively (chosen for the output vector to replicate the sensors used in the basic classical nulling 


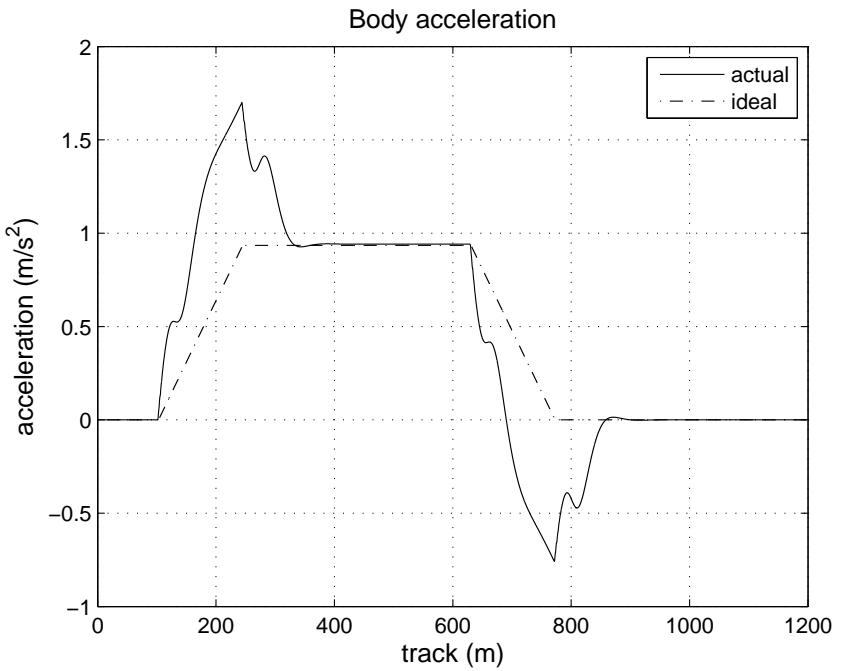

(a) Lateral acceleration

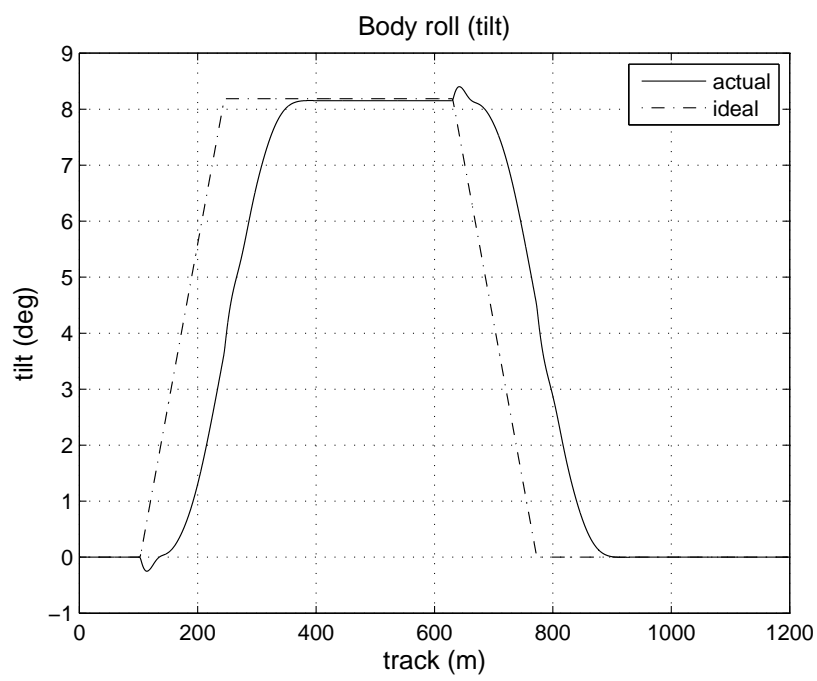

(b) Tilt angle

Fig. 23. Lateral acceleration and tilt profile on curved track with LQG

control). It is worth noting that the system transfer function with the two aforementioned measurements is not NMP.

It is very important to meet both deterministic (curve track) and stochastic (straight track) requirements, thus the following multi-objective optimisation problem was formulated 


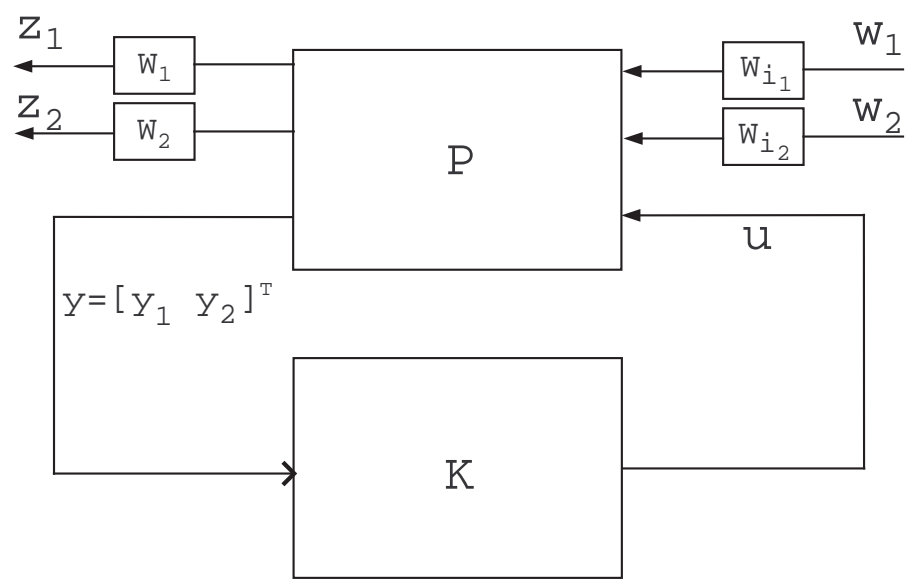

Fig. 24. Generalised Regulator configuration for multi-objective control

$$
\min _{K \in \mathbb{S}} \alpha\left\|W_{1} T_{z_{1} w}\right\|_{\infty}^{2}+\beta\left\|W_{2} T_{z_{2} w}\right\|_{2}^{2}
$$

in which $\mathbb{S}$ denotes the set of all internally stabilising controllers. The first regulated output $z_{1}$ for infinity-norm minimisation, was chosen as the effective cant deficiency $z_{1}=\theta_{d m}^{\prime}$. For the minimisation of the 2-norm, $z_{2}$ was chosen as the control input $u$ denoting the actuator roll angle $\delta_{a}$. Regulating $z_{1}$ to zero corresponds to $60 \%$ tilt compensation and thus attains the desired (steadystate) level of acceleration on steady state curve. $T_{z_{i} w}(i=1,2)$ denotes the (closed-loop) transfer functions between signals $w$ and $z_{1}, z_{2}$ respectively.

Multi-objective optimisation typically refers to the joint optimisation of a vector consisting of two or more functions, typically representing conflicting objectives. Common types of multi-objective optimisation problems include "Pareto-optimal" (non-inferior) optimality criteria, minimax optimality criteria, etc. In the context of this exercise, the term "multi-objective" refers simply to the fact that the cost function of the optimisation problem involves two different types of norms, capturing the deterministic and stochastic objectives of the design. The two different norms that are used here are the 2-norm and the infinity-norm. Thus, typical examples of multi-objective problems in our context include:

1. Constrained minimisation:

Minimise $\left\|W_{1} T_{x y}\right\|_{2}$ subject to $\left\|W_{2} T_{z w}\right\|_{\infty}<\gamma$,

2. Unconstrained minimisation:

Minimise $\beta\left\|W_{1} T_{x y}\right\|_{2}+\alpha\left\|W_{2} T_{z w}\right\|_{\infty}$, and

3. Feasibility problem:

Find a stabilising $K(s)$ (if one exists) such that $\left\|W_{1} T_{x y}\right\|_{2} \leq \gamma_{1}$ and $\left\|W_{2} T_{z w}\right\|_{\infty} \leq \gamma_{2}$ 
$T_{x y}$ and $T_{z w}$ represent two general closed-loop transfer functions, weighted via $W_{1}$ and $W_{2}$.

Scalars $\alpha$ and $\beta$, in (31), are positive definite design parameters which may be used to shift the emphasis of the optimisation problem between the minimisation of the $\left\|T_{z_{1} w}\right\|_{\infty}$ term (deterministic objective) and the $\left\|T_{z_{2} w}\right\|_{2}$ term (stochastic objective). The frequency-domain weights $W_{1}$ and $W_{2}$ have been chosen as:

$$
\begin{aligned}
& W_{1}(s)=10^{4} \frac{\frac{s}{200}+1}{\frac{s}{0.0001}+1} \\
& W_{2}(s)=0.5 \frac{s^{3}+1.59 s^{2}+0.58 s+0.06}{s^{3}+13.81 s^{2}+38.4 s+2.98}
\end{aligned}
$$

$W_{1}$ is essentially a low-pass filter with a very low pole cut-off frequency $\left(10^{-4} \frac{\mathrm{rads}}{\mathrm{s}}\right)$ and high gain at low frequencies, Fig. 25(a). Thus $W_{1}$ emphasises minimisation of the $\left\|T_{z_{1} w}\right\|_{\infty}$ term in the low frequency range and effectively enforces integral control on the regulated output $\left(z_{1}\right) . W_{2}$ is a high-pass filter with pole $\left(10 \frac{\mathrm{rads}}{\mathrm{s}}\right)$ and zero $\left(0.2 \frac{\mathrm{rads}}{\mathrm{s}}\right)$ cut-off frequencies. A lead/lag network is also included in $W_{2}$, in the range of $\left[.1 \frac{\mathrm{rads}}{\mathrm{s}}, 6 \frac{\mathrm{rads}}{\mathrm{s}}\right]$, which found to have a positive effect on controller design (by enhancing the cross-over frequency of $W_{1}, W_{2}$ ), Fig. 25(a). By limiting the high-frequency components of the control input $\left(z_{2}\right)$, effectively places a limit on the closed-loop bandwidth of the system, which in turn limits the RMS acceleration on straight track (stochastic case). Additional benefits include a smoother control signal and improved robustness properties of the controller when the effects of uncertainty in $P(s)$ and in the actuator dynamics are taken into account. Moreover, the relative weighting between $w_{1}$ and $w_{2}$ were simply set to unity, i.e.

$$
W_{i}=\left[\begin{array}{cc}
W_{i_{1}} & 0 \\
0 & W_{i_{2}}
\end{array}\right]=\left[\begin{array}{ll}
1 & 0 \\
0 & 1
\end{array}\right]
$$

Thus the energy of either of the signals is equally incorporated in the cost function. Increasing either $W_{i_{1}}$ or $W_{i_{2}}$ with respect to the other will put more emphasis on the deterministic or the stochastic track respectively. However, the current choice of $W_{i}$ provides the best results.

The minimisation problem in (31) was solved in Matlab using the LMI toolbox [14], i.e. representing the problem in a set of Linear Matrix Inequalities and follow a convex optimisation approach. This technique has very attractive computational properties and is widely used in systems and control theory.

For controller design, the generalised plant was formulated as follows

$$
\begin{aligned}
\dot{x} & =A x+B_{1} w+B_{2} u \\
z_{\infty} & =C_{\infty} x+D_{\infty 1} w+D_{\infty 2} u \\
z_{2} & =C_{2} x+D_{21} w+D_{22} u \\
y & =C_{y} x+D_{y 1} w
\end{aligned}
$$




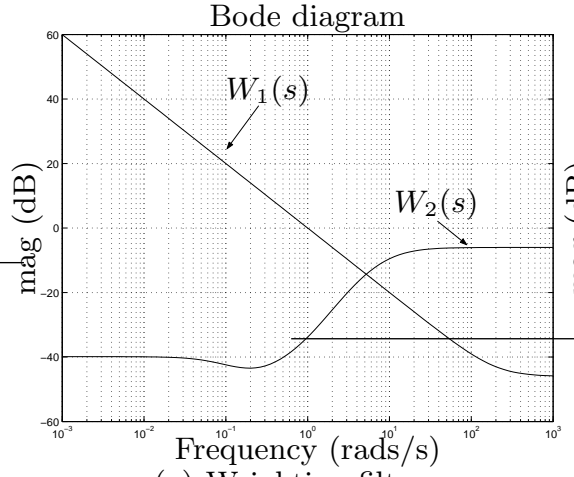

(a) Weighting filters

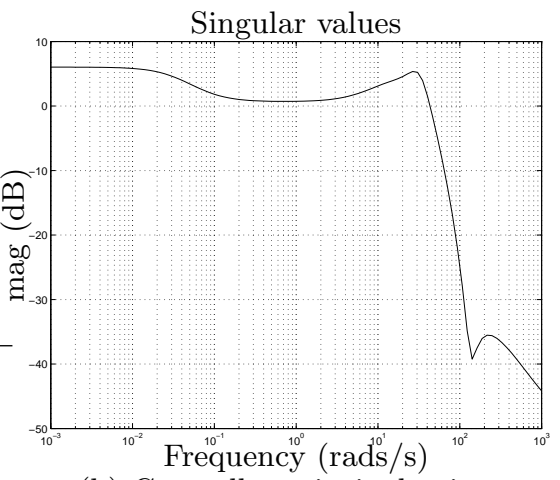

(b) Controller principal gains

Fig. 25. Multi-objective $H_{\infty} / H_{2}$ LMI approach scheme

where all matrices can be formed based upon the state space model of the system and the specifications in the generalised plant discusses earlier in this section. The controller can be then found by using matlab function hinfmix(). The optimisation problem was solved for a few combinations of the $\alpha$ and $\beta$

Table 6. $\alpha-\beta$ combinations for the $H_{\infty} / H_{2}$ problem

\begin{tabular}{llcc}
\hline$\alpha$ & $\beta$ & Ride Quality-Degrad.(\%) & Deviations-Determ.(\%g) \\
\hline 1 & 1 & 21.7 & 1.95 \\
1 & 2.5 & 10 & 2.15 \\
1 & 5 & 4.95 & 2.37 \\
1 & 10 & 3.4 & 2.62 \\
1 & 20 & 2.1 & 2.9 \\
\hline
\end{tabular}

Ride Quality-Degrad.: ride-quality degradation @ $58 \mathrm{~m} / \mathrm{s}$ of active system compared to passive @ $58 \mathrm{~m} / \mathrm{s}$ (straight track)

Deviations-Determ.: RMS acceleration deviation from the ideal response of an ideal tilting controller @ $58 \mathrm{~m} / \mathrm{s}$ (curved track)

parameters and the results can be seen in Table 6 . The results shown in the table clearly illustrate the fundamental trade-off between the deterministic and the stochastic objectives of the design.

As expected, increasing the value of $\beta$ relative to $\alpha$ places more emphasis on the stochastic aspects of the design, and as a result the RMS acceleration on straight track is further reduced. This is at the expense of deterministic performance and, therefore, the curved track response becomes slower (larger deviations from the ideal tilt response). Since it is required that stochastic performance deteriorates by no more than $7.5 \%$ compared to the passive system, the "best" design was obtained for $\alpha=1$ and $\beta=5$. The result returned in Matlab for the "best" configuration is shown below (summary) 


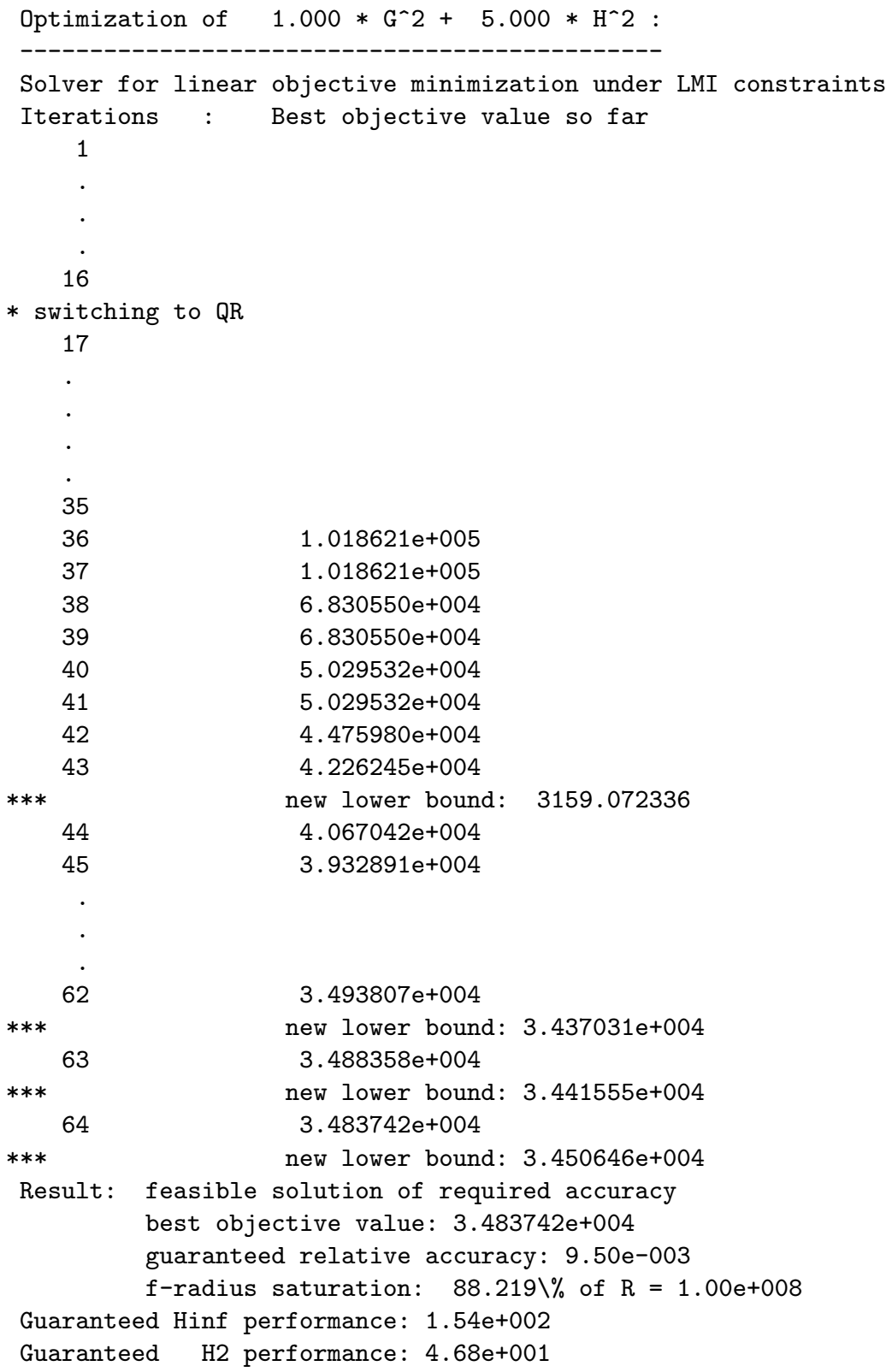

Note that, in the first few iterations, the algorithm does not find any solutions, however the solution converges soon after. The resulting controller is of 2-input/1output dimension due to the two measurements used in the formulation. The singular value plot is shown in Fig. 25(b), by definition depicting the largest singular of the two output/one input system transfer function. Note that the controller order is equal to 13 , i.e. 9 states from the train model +4 states from the weights. However, 
it can be easily reduced down to a 7 th order equivalent, e.g. via balanced truncation [10], with minimal degradation in performance.

The performance of the designed system is assessed in Table 7, where it can be seen that it is significantly improved compared to the classical nulling and LQG nulling-type control schemes. This exercises illustrate the usability of employing two measurements (compared to only one in the LQG scheme) and the effectiveness of distinguishing the design objectives in the cost function. For completeness the associated time history analysis for the design track is presented in Fig. 26.

Table 7. $H_{\infty} / H_{2}$ multi-objective LMI approach @ 58(m/s)

\begin{tabular}{llc}
\hline \multicolumn{3}{c}{ DETERMINISTIC } \\
\hline Lateral accel. & - steady-state & $9.53(\% \mathrm{~g})$ \\
(actual vs ideal) & - R.M.S. deviation error & $2.37(\% \mathrm{~g})$ \\
& - peak value & $13.66(\% \mathrm{~g})$ \\
Roll gyroscope & - R.M.S. deviation & $0.023(\mathrm{rad} / \mathrm{s})$ \\
& - peak value & $0.101(\mathrm{rad} / \mathrm{s})$ \\
(PCT-factor) & - peak jerk level & $7.07(\% \mathrm{~g} / \mathrm{s})$ \\
& - standing & $51.7(\%$ of passng) \\
& - seated & $14.93(\%$ of passng) \\
\hline \multicolumn{3}{c}{ STOCHASTIC } \\
\hline Passenger comfort - R.M.S. passive (equiv.) & $3.78(\% \mathrm{~g})$ \\
& - R.M.S. active & $3.96(\% \mathrm{~g})$ \\
& - degradation & $4.95(\%)$ \\
\hline
\end{tabular}

\subsection{Case Study Remarks}

This exercise has considered the design of local tilt controllers (a form of secondary railway suspension control) based upon advanced control concepts. The problems with early-type classical nulling approaches has been presented, and briefly discussed the currently-used precedence strategy. It has been shown that by using modern control methods, i.e. LQG and $H_{\infty}$ based schemes, the performance of nullingtype controllers can be significantly improved. Problems with the two model-based schemes include high-order controller size and the choice of weighting functions. Controller reduction can be employed for the former, while the latter requires a realistic setup of the design problem (to reduce the complexity of choosing the structure of the weights) and usually designer experience (this being the case for the majority of engineering applications). 


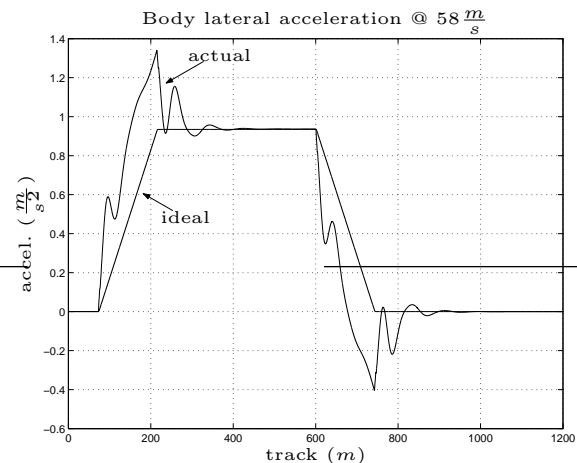

(a) Passenger acceleration

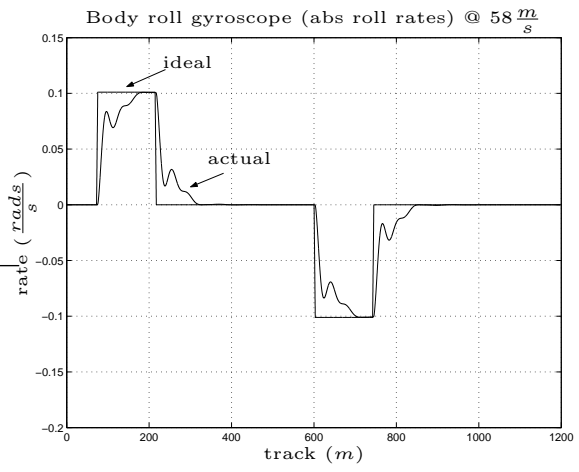

(b) Body Roll rate

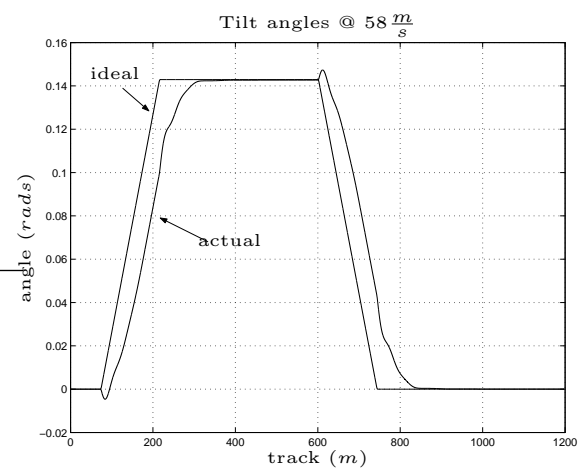

(c) Tilt angles

Fig. 26. Design track time history results for $H_{\infty} / H_{2}$ design

\section{References}

1. Iwnicki S (Ed.) (2006) Handbook of Railway Vehicle Dynamics. Taylor and Francis

2. Wickens A (2003) Fundamentals of Rail Vehicle Dynamics. Swets and Zeitlinger

3. Goodall RM, Brown S (2001) Tilt technology still evolving as the cost falls. Railway Gazette International. 521-525

4. Goodall, RM (1999) Tilting Trains and Beyond - the Future for Active Railway Suspensions: Part 1 Improving Passenger Comfort. Computing and Control Engineering Journal. August 153-159

5. Goodall RM, Bruni S, Mei TX (2005) Concepts and prospects for activelycontrolled railway running gear. Procs 19th IAVSD Symposium. Milano

6. Pearson JT, Goodall RM, Pratt I (1998) Control System Studies of an Active Anti-Roll Bar Tilt System for Railway Vehicles. Proceedings of the Institution of Mechanical Engineers Part F. 212(F1):43-60

7. Goodall RM, Zolotas AC, Evans J (2000) Assessment of the Performance of Tilt System Controllers. Proceedings of the Railway Technology Conference IMechE. C580/028/2000. Birmingham UK. November 231-239 
8. Maciejowski JM (1989) Multivariable Feedback Design. Addison-Wesley

9. Zhou K, Doyle JC (1998) Essentials of Robust Control. Prentice-Hall

10. Skogestad S, Postlethwaite I (2000) Multivariable Feedback Control: Analysis and Desgn. Wiley and Sons

11. Zolotas AC, Halikias GD, Goodall RM and Wang J (2006) Model Reduction Studies in LQG Optimal Control Design for High-Speed Tilting Railway Carriages. Proceedings of the 2006 American Control Conference. Minneapolis MN USA. June 1796-1801

12. Zolotas A C (2002) Advanced Control Strategies for Tilting Trains. PhD Thesis, Loughborough University, UK

13. Zolotas AC, Goodall RM (2005) Improving the tilt control performance of highspeed railway vehicles: an LQG approach. Proceedings of the 16th IFAC World Congress. Prague

14. Gahinet P, Nemirovski A, Laub A, Chilali M (1994) LMI Control Toolbox. Natick, MA, The MathWorks.

15. Zolotas AC, Halikias GD, Goodall RM (2000) A Comparison of Tilt Control Approaches for High Speed Railway Vehicles. Proceedings of the 14th International Conference on Systems Engineering ICSE 2000, Coventry UK. 2:632-636 


\section{Appendix A- Tilting Train Parameter values and notation}

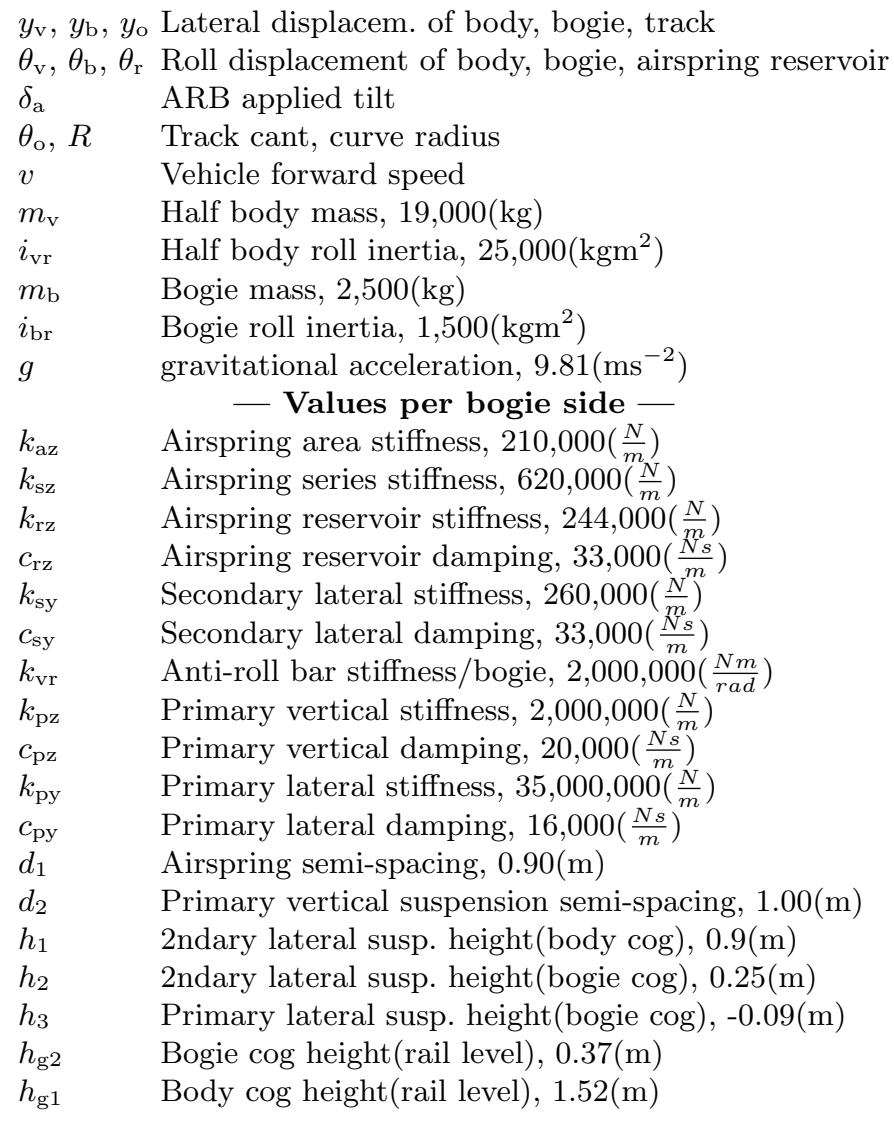

\section{Appendix B- $H_{\infty}$ based controllers: Preliminaries}

\section{Basic Notation}

A continuous time, linear time invariant, state space system is given by

$$
\begin{aligned}
& \dot{x}(t)=A x(t)+B u(t) \\
& y(t)=C x(t)+D u(t)
\end{aligned}
$$

where $A \in \Re^{n \times n}, B \in \Re^{n \times m}, C \in \Re^{p \times n}$ and $D \in \Re^{p \times m}$. The above state space system is characterised by the following transfer function with dimension $p \times n$ 


$$
G(s)=C(s I-A)^{-1} B+D
$$

This thesis adopts the following conventional state-space representation to represent $G(s)$

$$
G(s) \stackrel{s}{=}\left[\frac{A \mid B}{C \mid D}\right]
$$

Note also that the complex conjugate of $G(s)$ is given by

$$
G^{*}(s)=G^{T}(-s) \stackrel{s}{=}\left[\begin{array}{c|c}
-A^{T} & -B^{T} \\
\hline C^{T} & D^{T}
\end{array}\right]
$$

and if $G(s) G^{*}(s)=I=G^{*}(s) G(s)$ for all $s \in j \Re$, then $G(s)$ is said to be all-pass.

Finally if matrix $D$ is invertible, then $G^{-1}(s)$ is given by ${ }^{3}$

$$
G^{-1}(s) \stackrel{s}{=}\left[\begin{array}{c|c}
A-B D^{-1} C & B D^{-1} \\
\hline-D^{-1} C & D^{-1}
\end{array}\right]
$$

\section{- Frequency Domain Spaces and Norms}

This part introduces the meaning of frequency domain spaces and norms of real rational, matrix valued, transfer functions. For a more comprehensive study the reader is referred to Zhou and Doyle [9].

Let $\mathcal{R}$ denote the space of all real rational transfer function matrices. The $L_{2} / H_{2}$ norm of $G(s)$ is given by

$$
\|G\|_{2} \triangleq \sqrt{\frac{1}{2 \pi} \int_{-\infty}^{\infty} \operatorname{tr}\left(G^{*}(j \omega) G(j \omega)\right) d \omega}
$$

which is used to define the following spaces

(i). $\mathcal{R} \mathcal{L}_{2}$ refers to the space of all real rational transfer function matrices with no poles on the imaginary axis and is characterised by a finite $L_{2}$ norm.

(ii). $\mathcal{R} \mathcal{H}_{2}$ defines the space of all transfer function matrices in $\mathcal{R} \mathcal{L}_{2}$ with no poles in $\operatorname{Re}(s)>0$.

The $L_{\infty} / H_{\infty}$ norm of $G(s)$ is given by

$$
\|G\|_{\infty} \triangleq \sup _{\omega \in \Re} \bar{\sigma}[G(j \omega)]
$$

and

(i). $\mathcal{R} \mathcal{L}_{\infty}$ refers to the space of all real rational transfer function matrices with no poles on the imaginary axis (with finite $L_{\infty}$ norm).

(ii). $\mathcal{R} \mathcal{H}_{\infty}$ defines the space of all transfer function matrices in $\mathcal{R} \mathcal{L}_{\infty}$ with no poles in $\operatorname{Re}(s)>0$.

\footnotetext{
${ }^{3}$ Using the matrix inversion lemma:

$$
\left(A_{1}+A_{2} A_{3} A_{4}\right)^{-1}=A_{1}^{-1}-A_{1}^{-1} A_{2}\left(A_{4} A_{1}^{-1} A_{2}+A_{3}^{-1}\right)^{-1} A_{4} A_{1}^{-1} .
$$
}


Also, the $H_{\infty}$ norm of a stable transfer function $G(s)$ is its largest input/output RMS gain

$$
\|G\|_{\infty} \triangleq \sup _{\substack{u \in L_{2} \\ u \neq 0}} \frac{\|y\|_{L_{2}}}{\|u\|_{L_{2}}}
$$

where $L_{2}$ is the space of signals having finite energy and $y$ is the output of the system $G$ for a given input $u$. Thus, for any input $u$ of unit energy, the output energy in $y$ is bounded by the $H_{\infty}$ norm of $G(s)$.

\section{- Linear Fractional Transformations}

The basic concept of Linear Fractional Transformations is outlined in this section. Linear Fractional Transformations (LFT) are frequently used in the area of $H_{\infty}$ optimisation as well as in other areas of control theory. They do represent a means of standardising a wide variety of feedback arrangements [10].

Let $P(s)$ define a transfer function matrix with the following state-space representation

$$
P(s) \stackrel{s}{=}\left[\begin{array}{l|ll}
A & B_{1} & B_{2} \\
\hline C_{1} & D_{11} & D_{12} \\
C_{2} & D_{21} & D_{22}
\end{array}\right]
$$

which can be also partitioned as

$$
P(s) \stackrel{s}{=}\left[\begin{array}{l|l}
P_{11} & P_{12} \\
\hline P_{21} & P_{22}
\end{array}\right]
$$

where

$$
P_{i j}(s)=C_{i}(s I-A)^{-1} B_{j}+D_{i j}
$$

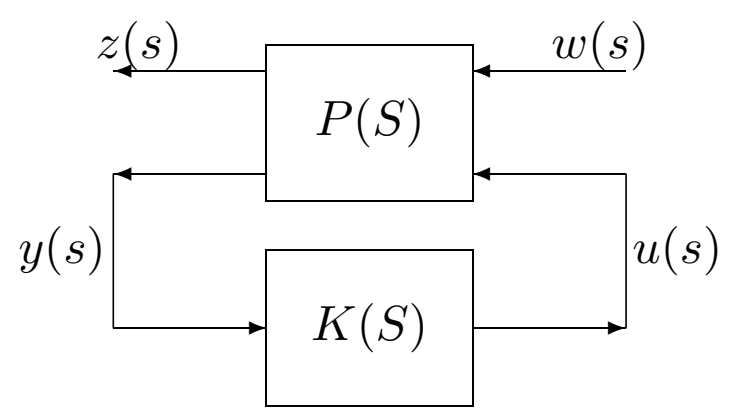

Fig. 27. The Generalised Regulator Configuration

Referring to Fig. 27, which presents the generalised regulator configuration, the (lower) linear fractional transformation ${ }^{4}$ of $P$ and $K$ is given by

$$
\mathcal{F}_{L}(P, K) \triangleq P_{11}+P_{12} K\left(I-P_{22} K\right)^{-1} P_{21}
$$

\footnotetext{
${ }^{4}$ There is also the concept of the upper LFT which is employed in representing uncertainties in a system [10].
} 
for $\operatorname{det}\left(I-P_{22} K\right) \neq 0 . P(s)$ represents the "generalised plant", consisting of the nominal model $G(s)$ combined with all frequency weightings appropriately chosen to shift the emphasis with frequency between different design objectives. In addition, the signals are: $u$ the control variables, $w$ the exogenous inputs such as disturbances $w_{d}$ and commands $r, y$ the measured variables and $z$ the regulated outputs, i.e. the signals need to minimise to meet the design objectives. In fact $\mathcal{F}_{L}(P, K)$ represents the transfer function between $w$ and $z$ in Fig. 27, i.e.

$$
z(s)=\left[P_{11}+P_{12} K\left(I-P_{22} K\right)^{-1} P_{21}\right] w(s)
$$

$H_{\infty}$ and $H_{2}$ control implies the minimisation of the $H_{\infty}$-norm and the $H_{2}$-norm of $\mathcal{F}_{L}(P, K)$ respectively. 